\title{
How Much FBMC/OQAM Is Better than FBMC/QAM? A Tentative Response Using the POPS Paradigm
}

\author{
Wafa Khrouf $(\mathbb{D}$, Mohamed Siala $(\mathbb{D}$, and Fatma Abdelkefi $(\mathbb{D}$ \\ Mediatron Laboratory, Higher School of Communications of Tunis (SUP'COM), 2083 Ariana, Tunisia \\ Correspondence should be addressed to Wafa Khrouf; wafa.khrouf@supcom.tn
}

Received 28 July 2017; Revised 3 November 2017; Accepted 20 December 2017; Published 10 April 2018

Academic Editor: Malte Schellmann

Copyright (c) 2018 Wafa Khrouf et al. This is an open access article distributed under the Creative Commons Attribution License, which permits unrestricted use, distribution, and reproduction in any medium, provided the original work is properly cited.

\begin{abstract}
A major trend of the current research in $5 \mathrm{G}$ is to find well time and frequency localized waveforms, dedicated to non-orthogonal wireless multi-carrier systems. The ping-pong optimized pulse shaping (POPS) paradigm was proposed as a powerful technique to generate a family of waveforms, ensuring an optimal signal to interference plus noise ratio (SINR) at the receiver. In this paper, we derive, for the first time, the analytical expression of the SINR for FBMC/OQAM systems. We then adopt the POPS algorithm in the design of optimum transmit and receive waveforms for FBMC/OQAM, with respect to the SINR criterion. For relatively high dispersions, numerical results show that the optimized waveforms provide a gain of $7 \mathrm{~dB}$, in terms of SINR, compared to the PHYDYAS waveform. They also show that the obtained waveforms offer better out-of-band (OOB) emissions with regard to those of the IOTA waveform. Furthermore, we notice that FBMC/OQAM systems present a gain of $4 \mathrm{~dB}$ in SINR, compared to FBMC/QAM systems, when both operate at their time-frequency lattice critical densities. However, FBMC/QAM systems can guarantee, with a reduced computational complexity, a comparable performance to FBMC/OQAM systems, in terms of SINR, when their spectral efficiency is relatively reduced by less than $5 \%$.
\end{abstract}

\section{Introduction}

Orthogonal frequency division multiplexing (OFDM) systems have witnessed a considerable interest in the last decade [1]. However, in their present form, they are deemed inapt of guaranteeing the required quality of service (QoS) in several new challenging applications brought by $5 \mathrm{G}$ systems. This inaptitude is due to many factors. Among them, we can cite the strong spectral leakage, which can only be controlled with strict frequency synchronization. As a consequence, any lack of perfect frequency synchronization causes important intercarrier interference (ICI). Besides, this incapacity is a result of the intersymbol interference (ISI), when the actual channel delay spread exceeds the cyclic prefix (CP) duration, and/or strict time synchronization is relaxed to save signalling resources and delays [2].

To overcome OFDM limitations and meet $5 \mathrm{G}$ requirements, several European projects have been launched recently, such as "mobile and wireless communications enablers for twenty-twenty (2020) information society" (METIS) [3], "flexible air interface for scalable service delivery within wireless communication networks of the 5th generation" (FANTASTIC5G) [4], "enhanced multi-carrier techniques for professional ad-hoc and cell-based communications" (EMPHATIC) [5], and "5th generation non-orthogonal waveforms for asynchronous signalling" (5GNOW) [6]. In the 5 GNOW project, for instance, various modulations have been suggested [7], namely, filter bank multi-carrier (FBMC), generalized frequency division multiplexing (GFDM), and universal filtered multi-carrier (UFMC). Among these multiple access techniques, FBMC seems to be a good candidate for $5 \mathrm{G}$ systems. In the literature, many researches shed light on the advantages of FBMC with offset quadrature amplitude modulation (FBMC/OQAM) systems compared to OFDM systems. Among these advantages, one can cite their robustness to channel time and frequency spreading [8], since their waveforms are well localized in time and frequency. However, FBMC/OQAM implementation and analytical derivation of the SINR are more complex than those of OFDM.

In this paper, we focus on FBMC/OQAM systems, which are also known as OFDM/OQAM and staggered multitone (SMT) [9]. They consist in transmitting separately and 
alternately, in each subcarrier, the in-phase and quadrature components of complex symbols used in quadrature amplitude modulation (QAM). We believe that there are a limited number of studies which have attempted to derive the SINR analytical expression of FBMC/OQAM systems [10-12]. In [10], the SINR is derived for known transmitter (Tx) and receiver $(\mathrm{Rx})$ waveforms. On the other hand, in [11], the authors calculate the signal to interference ratio (SIR) for a channel with a carrier frequency offset (CFO), whereas in [12], the authors derive the SIR for a channel with a $\mathrm{CFO}$ and/or a time offset (TO). In contrast, in this paper, we derive the analytical expression of the SINR for multicarrier transmissions in the case of highly time and frequency dispersive channels. Once the SINR analytical expression is established, we seek the appropriate Tx/Rx waveforms, which optimize the SINR. To this end, we extend the application of the POPS paradigm, adopted for FBMC/QAM systems in [13], to FBMC/OQAM systems. The POPS algorithm, firstly introduced in [14], has the merit of being very effective in the optimization of the transmit and receive waveforms. It is an iterative algorithm, which enables simple and offline optimization of the waveforms at the $\mathrm{Tx} / \mathrm{Rx}$ sides, by maximizing the SINR.

The optimal $\mathrm{Tx} / \mathrm{Rx}$ waveforms, resulting from POPS, are robust against the ISI and ICI incurred by mobile radio communication propagation channels. However, the imaginary part of the interference, known as intrinsic interference $[15,16]$, is inherent to FBMC/OQAM systems. To account for it, channel estimation is always required. The intrinsic interference, remaining after channel estimation, cannot be neglected unless the channel is not severely dispersive in single-input single-output (SISO) systems, or linear spatial equalization schemes are used in moderately dispersive channels in multiple-input multiple-output (MIMO) systems. Moreover, if spatial equalization other than linear schemes is used in MIMO, a complex equalization process is required to account for the intrinsic interference. Keeping this in mind, we exclusively focus, in this paper, on SISO systems and target the optimization of $\mathrm{Tx} / \mathrm{Rx}$ waveforms in this context.

Our main contributions in this paper are the following:

(i) We provide an analytical expression of the SINR, for FBMC/OQAM systems, in arbitrary channel conditions, for whatever $\mathrm{Tx} / \mathrm{Rx}$ waveforms.

(ii) We compare, theoretically, the performances of FBMC/QAM and FBMC/OQAM systems in an arbitrary propagation framework.

(iii) We present and detail the performance of FBMC/ QAM systems with lattice densities below or equal to 1.

(iv) We quantify potential gains that can be realized by FBMC/OQAM systems with respect to FBMC/QAM systems, using identical and/or different lattice densities.

(v) We compare the performances of POPS waveforms with respect to physical layer for dynamic access and cognitive radio (PHYDYAS) and isotropic orthogonal transform algorithm (IOTA) waveforms, in FBMC/ OQAM systems.

This paper is organized as follows. In Section 3, we present the adopted system model for FBMC/OQAM and FBMC/QAM systems. Then, we focus on the derivation of the useful, interference, and noise powers in Section 4 and derive the SINR expression for FBMC/OQAM systems in Section 5. In Section 6, we describe the POPS algorithm suitable for the design of optimal waveforms. We dedicate Section 7 to the illustration of the obtained analytical results. Finally, we present conclusion and perspectives to this work in Section 8 .

\section{Notations}

Boldface lower and upper case letters refer to vectors and matrices, respectively, and $M_{k l}$ refers interchangeably to the $(p, q)^{\text {th }}$ element of the matrix $M$. The superscripts $\cdot^{*}, \cdot^{T}$, and .${ }^{-1}$ denote the conjugate of a function, the transpose of a vector, and the inverse of a matrix, respectively. We denote by $\mathbb{E}[\cdot]$ the expectation operator, by $\Re\{\cdot\}$ the real-part operator, by $|\cdot|$ the absolute value, and by $\llbracket n, m \rrbracket$ the set of integers between $n$ and $m$, where $n \leq m$. We denote by $\langle x, y\rangle=$ $\int x^{*}(t) y(t) d t$ the Hermitian scalar product, by $\langle x, y\rangle_{\Re}=$ $\mathfrak{R}\{\langle x, y\rangle\}$ the real scalar product, and by $\|x\|=\sqrt{\langle x, x\rangle}=$ $\sqrt{\langle x, x\rangle_{\Re}}=\sqrt{\int|x(t)|^{2} d t}$ the norm of $x$.

\section{System Model}

In this section, we consider a general FBMC system model in its continuous-time version. We denote by $T$ the FBMC (QAM or OQAM) symbol period, by $F$ the frequency separation between adjacent subcarriers, by $F T$ the time-frequency occupancy of each transmitted symbol, and by $\Delta=1 / F T$ the lattice density. To preserve the same spectral efficiency as in FBMC/QAM, FBMC/OQAM should use twice the lattice density, since real symbols are transmitted in place of complex symbols. Hence, to have a critical density, the lattice density should be equal to $\Delta=2$ in FBMC/OQAM, when $\Delta=1$ in FBMC/QAM.

The FBMC transmitted signal can be written under the following expression

$$
e(t)=\sum_{m n} a_{m n} \varphi_{m n}(t)
$$

where $m, n \in \mathbb{Z}, a_{m n}$ is the data symbol transmitted at time $n T$ and frequency $m F$, and

$$
\varphi_{m n}(t)=e^{j \theta_{m n}} \varphi(t-n T) e^{j 2 \pi m F t}
$$

refers to the phase, time, and frequency shifted version of the transmitter prototype waveform, $\varphi(t)$, used to transmit a symbol $a_{m n}$. The phase shift $\theta_{m n}$ is used in FBMC/OQAM to guarantee the orthogonality between the in-phase and quadrature phase components of the complex symbols used in FBMC/QAM, with respect to the real scalar product $\langle\cdot, \cdot\rangle_{\mathfrak{R}}$. In practice, we take $\theta_{m n}=(m+n) \pi / 2$, for FBMC/OQAM systems, and $\theta_{m n}=0$, for FBMC/QAM systems. 
The received signal is given by

$$
r(t)=\sum_{m n} a_{m n} \tilde{\varphi}_{m n}(t)+n(t),
$$

where $\widetilde{\varphi}_{m n}(t)=\int c(\tau ; t) \varphi_{m n}(t-\tau) d \tau$ is the channel-distorted version of $\varphi_{m n}(t), c(\tau ; t)$ is the channel impulse response (CIR) at time $t$, and $n(t)$ is a base-band complex additive white Gaussian noise (AWGN) with zero mean and two-sided power spectral density (PSD), $N_{0}$. For simplification reasons, we consider a channel with a finite number of paths, $L$, and a CIR equal to $c(\tau ; t)=\sum_{l=0}^{L-1} c_{l} e^{j 2 \pi v_{l} t} \delta\left(\tau-\tau_{l}\right)$, where $c_{l}, v_{l}$, and $\tau_{l}$ are, respectively, the amplitude, frequency Doppler shift, and time delay shift of the $l^{\text {th }}$ path. The paths amplitudes $c_{l}$, $l=0, \ldots, L-1$, are assumed to be centered and uncorrelated random complex Gaussian variables with average powers $\pi_{l}=\mathbb{E}\left[\left|c_{l}\right|^{2}\right]$.

\section{Useful, Interference, and Noise Powers}

In this section, we evaluate the useful, interference, and noise powers of both FBMC/OQAM and FBMC/QAM systems, using the same propagation channel conditions.

4.1. FBMC/OQAM System. In FBMC/OQAM systems, decision variables are calculated using a real scalar product. Therefore, in nondispersive channels, where the interference is purely imaginary, perfect orthogonality is achieved. Unfortunately, in the more general case of dispersive channels, as treated in this paper, the real part of the interference becomes an integral part of the decision variable on symbol $a_{k l}$, in (1), which is given by

$$
\Lambda_{k l}=\left\langle e^{j \chi_{k l}} \psi_{k l}, r\right\rangle_{\mathfrak{R}},
$$

where $\psi_{k l}(t)=e^{j \theta_{k l}} \psi(t-l T) e^{j 2 \pi k F t}$ is the phase, time, and frequency shifted version of the receiver prototype waveform, $\psi(t)$, used for the demodulation of the real symbol $a_{k l}$, and the phase $\chi_{k l}$ is used to compensate, even partially, the phase shift incurred by the channel at the time-frequency position, $(l T, k F)$, occupied by $a_{k l}$. Choosing a phase shift for $\psi_{k l}(t)$, equal to $\theta_{k l}=(k+l) \pi / 2$, guarantees a quasi-orthogonality between the alternately transmitted in-phase and quadrature phase components of FBMC/QAM, whatever the considered real symbol, $a_{k l}$, to be demodulated. Therefore, the decision variables characteristics are invariant by time and frequency translations within the time-frequency lattice, keeping the time-frequency lattice unchanged. Making this necessary assumption, we can, without loss of generality, focus on the evaluation of the SINR for symbol $a_{00}$. The decision variable on $a_{00}$ can be expanded into three terms, as

$$
\begin{aligned}
\Lambda_{00}= & \underbrace{a_{00}\left\langle e^{j \chi_{00}} \psi_{00}, \widetilde{\varphi}_{00}\right\rangle_{\mathfrak{R}}}_{U_{00}} \\
& +\underbrace{\sum_{\substack{m) \\
(m) \neq(0,0)}} a_{m n}\left\langle e^{j \chi_{00}} \psi_{00}, \widetilde{\varphi}_{m n}\right\rangle_{\mathfrak{R}}}_{I_{00}} \\
& +\underbrace{\left\langle e^{j \chi_{00}} \psi_{00}, n\right\rangle_{\mathfrak{R}}}_{N_{00}},
\end{aligned}
$$

where $U_{00}, I_{00}$, and $N_{00}$ are the useful, interference, and noise terms, respectively.

Conditional on a given realization of the CIR, $c(\tau ; t)$, the average powers of the useful and interference terms are expressed as

$$
\begin{aligned}
P_{U}^{c} & =\mathbb{E}\left[U_{00}^{2}\right]=\mathbb{E}\left[a_{00}^{2}\right]\left(\Re\left\{e^{-j \chi_{00}}\left\langle\psi_{00}, \widetilde{\varphi}_{00}\right\rangle\right\}\right)^{2}, \\
P_{I}^{c} & =\mathbb{E}\left[I_{00}^{2}\right] \\
& =\mathbb{E}\left[a_{m n}^{2}\right] \sum_{\substack{m n \\
(m, n) \neq(0,0)}}\left(\Re\left\{e^{-j \chi_{00}}\left\langle\psi_{00}, \tilde{\varphi}_{m n}\right\rangle\right\}\right)^{2} .
\end{aligned}
$$

The expression in (7) results from the uncorrelated nature of the real transmitted symbols, $a_{m n}$. The average transmitted energy corresponding to symbol $a_{m n}$ is given by $E_{s}=$ $\mathbb{E}\left[\left(a_{m n}\|\varphi\|\right)^{2}\right]=\mathbb{E}\left[a_{m n}^{2}\right]\|\varphi\|^{2}$. Let $E$ be the mean energy of a complex symbol in the case of FBMC/QAM systems, then, for comparison purposes, we assume that $E_{s}=E / 2$, which means that $\mathbb{E}\left[a_{m n}^{2}\right]=E_{s} /\|\varphi\|^{2}=E / 2\|\varphi\|^{2}$.

To calculate the SINR, an averaging over the channel realizations is needed. This step is precisely the most challenging and complicated step in FBMC/OQAM systems, since, in addition to $\widetilde{\varphi}_{m n}(t)$, the phase compensation term, $e^{j \chi_{00}}$, is also dependent on the same channel realizations. For an optimum choice of the compensation factor maximizing the SINR, we use

$$
e^{j \chi_{00}}=\frac{\left\langle\psi_{00}, \widetilde{\varphi}_{00}\right\rangle}{\left|\left\langle\psi_{00}, \widetilde{\varphi}_{00}\right\rangle\right|},
$$

which captures the phase shift incurred by the channel realization on the decision variable of symbol $a_{00}$, prior to casting to the real part. This is the ideal choice of $\chi_{00}$ that allows a compensation with the exact phase shift experienced by the symbol, which is caused by the channel. However, this choice makes the optimization step intractable, since the expected form of the SINR will not be a generalized Rayleigh quotient, but the ratio of two quaternary forms on each of the searched transmitter and receiver waveforms. For a further simplification of the optimization problem, with an expected tractable form of the SINR, we can use the transfer function of the channel, $C(f ; t)=\sum_{l=0}^{L-1} c_{l} e^{j 2 \pi v_{l} t} e^{-j 2 \pi \tau_{l} f}$, which is the Fourier transform of the CIR, $c(\tau ; t)$, with respect to $\tau$, and use the compensation factor,

$$
e^{j \chi_{00}}=\frac{C(0 ; 0)}{|C(0 ; 0)|}=\frac{\sum_{l=0}^{L-1} c_{l}}{\left|\sum_{l=0}^{L-1} c_{l}\right|} .
$$

By averaging expressions (6) and (7) on the realizations of the channel, the useful and interference powers are, respectively, given by

$$
\begin{aligned}
P_{U} & =\mathbb{E}\left[P_{U}^{c}\right]=\frac{E}{2\|\varphi\|^{2}} \mathbb{E}\left[\left(\mathfrak{R}\left\{e^{-j \chi_{00}}\left\langle\psi_{00}, \widetilde{\varphi}_{00}\right\rangle\right\}\right)^{2}\right], \\
P_{I} & =\mathbb{E}\left[P_{I}^{c}\right] \\
& =\frac{E}{2\|\varphi\|^{2}} \sum_{\substack{m n n \\
(m, n) \neq(0,0)}} \mathbb{E}\left[\left(\Re\left\{e^{-j \chi_{00}}\left\langle\psi_{00}, \widetilde{\varphi}_{m n}\right\rangle\right\}\right)^{2}\right] .
\end{aligned}
$$


Let $\bar{E}=E \sum_{l=0}^{L-1} \pi_{l}$ be the average energy received per complex symbol and let $\tilde{\pi}_{l}=\pi_{l} / \sum_{l=0}^{L-1} \pi_{l}$ be the normalized multipath power profile of the channel, with $\sum_{l=0}^{L-1} \tilde{\pi}_{l}=1$. Using the results of Appendix 1, we can write

$$
\begin{aligned}
P_{U}= & \frac{\bar{E}}{4\|\varphi\|^{2}}\left[\sum_{k=0}^{L-1} \tilde{\pi}_{k}\right. \\
& \cdot \iint \psi_{00}(t) \psi_{00}^{*}(s) \varphi_{00}^{*}\left(t-\tau_{k}\right) \varphi_{00}\left(s-\tau_{k}\right) e^{j 2 \pi v_{k}(s-t)} d t d s \\
& +\Re\left\{\sum_{k, l=0}^{L-1} \tilde{\pi}_{k} \tilde{\pi}_{l}\right. \\
& \left.\left.\cdot \iint \psi_{00}^{*}(t) \psi_{00}^{*}(s) \varphi_{00}\left(t-\tau_{k}\right) \varphi_{00}\left(s-\tau_{l}\right) e^{j 2 \pi\left(v_{k} t+\gamma_{l} s\right)} d t d s\right\}\right], \\
P_{I}= & \frac{\bar{E}}{4}\|\varphi\|^{2} \sum_{(m, n) \neq(0,0)}\left[\sum_{k=0}^{L-1} \tilde{\pi}_{k}\right. \\
& \cdot \iint \psi_{00}(t) \psi_{00}^{*}(s) \varphi_{m n}^{*}\left(t-\tau_{k}\right) \varphi_{m n}\left(s-\tau_{k}\right) e^{j 2 \pi v_{k}(s-t)} d t d s \\
& +\Re\left\{\sum_{k, l=0}^{L-1} \tilde{\pi}_{k} \tilde{\pi}_{l}\right. \\
& \left.\left.\cdot \iint \psi_{00}^{*}(t) \psi_{00}^{*}(s) \varphi_{m n}\left(t-\tau_{k}\right) \varphi_{m n}\left(s-\tau_{l}\right) e^{j 2 \pi\left(\nu_{k} t+\gamma_{l} s\right)} d t d s\right\}\right] .
\end{aligned}
$$

To extend the obtained results to more general diffuse channels, obeying the wide sense stationary uncorrelated scattering (WSSUS) property [17], we consider the asymptotic configuration, where $L \rightarrow+\infty$ in (12). Denoting by $S(\tau, \nu)$ the scattering function of the channel and by $\widetilde{S}(\tau, \nu)=$ $S(\tau, \nu) / \iint S(\tau, \nu) d \tau d \nu$ its normalized version, we can rewrite $P_{U}$ and $P_{I}$ in a more general form as

$$
\begin{aligned}
& P_{U}=\frac{\bar{E}}{4\|\varphi\|^{2}}\left[\int \int \widetilde { S } ( \tau , \nu ) \left(\iint \psi(t) \psi^{*}(s) \varphi^{*}(t-\tau)\right.\right. \\
& \left.\cdot \varphi(s-\tau) e^{j 2 \pi v(s-t)} d t d s\right) d \tau d v \\
& +\Re\left\{\iiint \widetilde{S}\left(\tau_{1}, v_{1}\right) \widetilde{S}\left(\tau_{2}, v_{2}\right)\right. \\
& \cdot\left(\iint \psi^{*}(t) \psi^{*}(s) \varphi\left(t-\tau_{1}\right)\right. \\
& \left.\left.\left.\cdot \varphi\left(s-\tau_{2}\right) e^{j 2 \pi\left(v_{1} t+v_{2} s\right)} d t d s\right) d \tau_{1} d \tau_{2} d \nu_{1} d v_{2}\right\}\right], \\
& P_{I}=\frac{\bar{E}}{4\|\varphi\|^{2}} \sum_{\substack{m n \\
(m, n) \neq(0,0)}}\left[\int \int \widetilde { S } ( \tau , \nu ) \left(\iint \psi(t) \psi^{*}(s) \varphi_{m n}^{*}(t-\tau)\right.\right. \\
& \left.\cdot \varphi_{m n}(s-\tau) e^{j 2 \pi \nu(s-t)} d t d s\right) d \tau d v \\
& +\Re\left\{\iiint \widetilde{S}\left(\tau_{1}, v_{1}\right) \widetilde{S}\left(\tau_{2}, v_{2}\right)\right. \\
& \cdot\left(\iint \psi^{*}(t) \psi^{*}(s) \varphi_{m n}\left(t-\tau_{1}\right)\right. \\
& \left.\left.\left.\cdot \varphi_{m n}\left(s-\tau_{2}\right) e^{j 2 \pi\left(v_{1} t+v_{2} s\right)} d t d s\right) d \tau_{1} d \tau_{2} d v_{1} d v_{2}\right\}\right] .
\end{aligned}
$$

The noise power is given by

$$
P_{N}=\mathbb{E}\left[\left(\Re\left\{e^{-j \chi_{00}}\left\langle\psi_{00}, n\right\rangle\right\}\right)^{2}\right],
$$

where $e^{-j \chi_{00}}\left\langle\psi_{00}, n\right\rangle$ is a circular random complex Gaussian variable, which is independent of $e^{-j \chi_{00}}$ and has the same variance as $\left\langle\psi_{00}, n\right\rangle$. Thus, $\mathfrak{R}\left\{e^{-j \chi_{00}}\left\langle\psi_{00}, n\right\rangle\right\}$ is a random real Gaussian variable, which has half the variance of $\left\langle\psi_{00}, n\right\rangle$. Accordingly,

$$
P_{N}=\frac{1}{2} \mathbb{E}\left[\left|\left\langle\psi_{00}, n\right\rangle\right|^{2}\right]
$$

Since the noise is white, with autocorrelation function $R_{n n}(\tau)=N_{0} \delta(\tau)$, where $\delta(\cdot)$ is the Dirac delta function, we can write

$$
\begin{aligned}
\mathbb{E} & {\left[\left|\left\langle\psi_{00}, n\right\rangle\right|^{2}\right] } \\
& =\mathbb{E}\left[\left(\int \psi_{00}^{*}(t) n(t) d t\right)^{*}\left(\int \psi_{00}^{*}(s) n(s) d t\right)\right] \\
& =\iint \psi_{00}(t) \psi_{00}^{*}(s) \mathbb{E}\left[n^{*}(t) n(s)\right] d t d s \\
& =N_{0}\left\|\psi_{00}\right\|^{2} .
\end{aligned}
$$

Consequently, the noise power can be expressed as

$$
P_{N}=\frac{N_{0}}{2}\|\psi\|^{2}
$$

4.2. FBMC/QAM System. The SINR expression for FBMC/ QAM systems was derived in $[13,14]$, in the case of continuous- and discrete time, respectively. In this section, we briefly present the main steps considered to find its analytical expression in the case of continuous signals.

The decision variable on complex symbol $a_{k l}$, bearing simultaneously both in-phase and quadrature phase components, uses the conventional Hermitian scalar product and has the following expression

$$
\Lambda_{k l}=\left\langle\psi_{k l}, r\right\rangle=\int \psi_{k l}^{*}(t) r(t) d t
$$

where $\psi_{k l}(t)=\psi(t-l T) e^{j 2 \pi k F t}$ is the time and frequency shifted version of the receiver prototype waveform, $\psi(t)$, used for the demodulation of the complex symbol $a_{k l}$.

Again and as before for FBMC/OQAM, we will evaluate, without loss of generality, the SINR for symbol $a_{00}$. The decision variable on $a_{00}$ can be expanded into three terms as

$$
\begin{aligned}
\Lambda_{00}= & \underbrace{a_{00}\left\langle\psi_{00}, \widetilde{\varphi}_{00}\right\rangle}_{U_{00}}+\underbrace{\sum_{\substack{m n \\
(m, n) \neq(0,0)}} a_{m n}\left\langle\psi_{00}, \widetilde{\varphi}_{m n}\right\rangle}_{I_{00}} \\
& +\underbrace{\left\langle\psi_{00}, n\right\rangle}_{N_{00}},
\end{aligned}
$$

where $U_{00}, I_{00}$, and $N_{00}$ are the useful, interference, and noise terms, respectively. 
Conditional on a given realization of the CIR, $c(\tau$; $t)$, the average powers of the useful and interference terms are given by

$$
\begin{aligned}
& P_{U}^{c}=\mathbb{E}\left[\left|U_{00}\right|^{2}\right]=\mathbb{E}\left[a_{00}^{2}\right]\left|\left\langle\psi_{00}, \widetilde{\varphi}_{00}\right\rangle\right|^{2}, \\
& P_{I}^{c}=\mathbb{E}\left[\left|I_{00}\right|^{2}\right]=\mathbb{E}\left[a_{m n}^{2}\right] \sum_{\substack{m n \\
(m, n) \neq(0,0)}}\left|\left\langle\psi_{00}, \widetilde{\varphi}_{m n}\right\rangle\right|^{2} .
\end{aligned}
$$

The expression in (23) results from the uncorrelated nature of the complex transmitted symbols, $a_{m n}$. Since the average transmitted energy of $a_{m n}$ is $E=\mathbb{E}\left[\left(a_{m n}\|\varphi\|\right)^{2}\right]=\mathbb{E}\left[a_{m n}^{2}\right]\|\varphi\|^{2}$, we conclude that $\mathbb{E}\left[a_{m n}^{2}\right]=E /\|\varphi\|^{2}$. By averaging the expressions in (22) and (23) on the realizations of the channel, we obtain

$$
\begin{aligned}
P_{U} & =\frac{E}{\|\varphi\|^{2}} \mathbb{E}\left[\left|\left\langle\psi_{00}, \widetilde{\varphi}_{00}\right\rangle\right|^{2}\right], \\
P_{I} & =\frac{E}{\|\varphi\|^{2}} \sum_{\substack{m n \\
(m, n) \neq(0,0)}} \mathbb{E}\left[\left|\left\langle\psi_{00}, \widetilde{\varphi}_{m n}\right\rangle\right|^{2}\right] .
\end{aligned}
$$

Using the same notations, $\bar{E}$ and $\tilde{\pi}_{l}$, as in FBMC/OQAM systems, the useful power can be written as

$$
\begin{aligned}
& P_{U}=\frac{\bar{E}}{\|\varphi\|^{2}} \sum_{k=0}^{L-1} \tilde{\pi}_{k} \iint \psi_{00}(t) \psi_{00}^{*}(s) \varphi_{00}^{*}\left(t-\tau_{k}\right) \\
& \cdot \varphi_{00}\left(s-\tau_{k}\right) e^{j 2 \pi v_{k}(s-t)} d t d s,
\end{aligned}
$$

and the interference power can be written as

$$
\begin{aligned}
P_{I} & =\frac{\bar{E}}{\|\varphi\|^{2}} \sum_{(m, n) \neq(0,0)} \sum_{k=0}^{L-1} \tilde{\pi}_{k} \iint \psi_{00}(t) \psi_{00}^{*}(s) \varphi_{m n}^{*}\left(t-\tau_{k}\right) \\
& \cdot \varphi_{m n}\left(s-\tau_{k}\right) e^{j 2 \pi v_{k}(s-t)} d t d s .
\end{aligned}
$$

As in FBMC/OQAM systems, to generalize the obtained results, we assume that we have a WSSUS channel and use the same normalized scattering function, $\widetilde{S}(\tau, \nu)$. Hence, $P_{U}$ and $P_{I}$ can, respectively, be expressed as

$$
\begin{aligned}
P_{U} & =\frac{\bar{E}}{\|\varphi\|^{2}} \iint \widetilde{S}(\tau, \nu) \iint \psi(t) \psi^{*}(s) \varphi^{*}(t-\tau) \varphi(s-\tau) \\
& \cdot e^{j 2 \pi \nu(s-t)} d t d s d \tau d \nu \\
P_{I} & =\frac{\bar{E}}{\|\varphi\|^{2}} \sum_{\substack{m n n \\
(m, n) \neq(0,0)}} \iint \widetilde{S}(\tau, \nu) \iint \psi(t) \psi^{*}(s) \varphi_{m n}^{*}(t-\tau) \\
& \cdot \varphi_{m n}(s-\tau) e^{j 2 \pi v(s-t)} d t d s d \tau d \nu .
\end{aligned}
$$

The noise power is given by

$$
P_{N}=\mathbb{E}\left[\left|\left\langle\psi_{00}, n\right\rangle\right|^{2}\right]=N_{0}\|\psi\|^{2} .
$$

\section{SINR Expression}

As can be noticed, we are trying to find the optimum continuous-time $\mathrm{Tx} / \mathrm{Rx}$ waveforms in the Hermitian space of square integrable functions, $L^{2}(\mathbb{R})$. Trying to directly find the best solutions in this space is not tractable numerically. One practical way to proceed is to explore the most pertinent finite subspace of $L^{2}(\mathbb{R})$, keeping in mind the nature of the optimization problem, which intuitively requires well-localized waveforms, both in time and in frequency. Therefore, we need to carefully choose an appropriate base of the exploration subspace used for expanding the searched solutions for the $\mathrm{Tx} / \mathrm{Rx}$ waveforms. One way to proceed is to use a finite subset of the well-known orthonormal base of Hermite functions, $\left\{h_{k}(t)\right\}_{k \in \mathbb{N}}$, which is an orthonormal base of $L^{2}(\mathbb{R})[18]$. One of the most important and desirable properties of these Hermite functions is that they provide, in decreasing order, the most localized functions in time and frequency. Hence, for the expansion on Hermite functions, we only need to keep the $K+1$ most localized Hermite functions in the representation of the sought optimum Tx/Rx waveforms. More precisely, we set

$$
\begin{aligned}
& \varphi(t)=\sum_{k=0}^{K} \alpha_{k} h_{k}(t), \\
& \psi(t)=\sum_{k=0}^{K} \beta_{k} h_{k}(t),
\end{aligned}
$$

where $\alpha_{k}, \beta_{k} \in \mathbb{R}$ and $h_{k}(t)=2^{1 / 4} 2^{-k / 2}(k !)^{-1 / 2} e^{-\pi t^{2}} H_{k}(t \sqrt{2 \pi})$ [19], with $H_{k}(t)$ being the Hermite polynomial of degree $k$. Then, we inject these expressions in (14) and (19), for FBMC/ OQAM systems, and in (28) and (30), for FBMC/QAM systems. Since the SINR is defined as SINR $=P_{U} /\left(P_{I}+P_{N}\right)$, we can write

$$
\begin{aligned}
\text { SINR } & =\sum_{k, l, p, q=0}^{K} \alpha_{p} \alpha_{q} \beta_{k} \beta_{l} M_{k l p q}^{(00)} \\
& \times\left(\sum_{k, l, p, q=0}^{K} \alpha_{p} \alpha_{q} \beta_{k} \beta_{l} \sum_{\substack{m n \\
(m, n) \neq(0,0)}} M_{k l p q}^{(m n)}\right. \\
& \left.+\left(1+\delta_{i}\right)\left(\frac{N_{0}}{\bar{E}}\right) \sum_{k, p=0}^{K} \alpha_{p}^{2} \beta_{k}^{2}\right)^{-1},
\end{aligned}
$$

where $\delta_{i}=1$ for FBMC/OQAM systems, $\delta_{i}=0$ for FBMC/ QAM systems, and

$$
\begin{aligned}
M_{k l p q}^{(m n)}= & \iint \widetilde{S}(\tau, v) A_{p k}(\tau+n T, v+m F) \\
& \cdot A_{q l}^{*}(\tau+n T, \nu+m F) d \tau d \nu \\
& +\delta_{i}(-1)^{m+n} \Re\left\{\left(\iint \widetilde{S}(\tau, \nu) A_{p k}\left(\tau_{1}+n T, \nu_{1}+m F\right)\right.\right. \\
& \left.\cdot e^{-j \pi\left(\tau_{1}+n T\right)\left(v_{1}-m F\right)} d \tau_{1} d \nu_{1}\right)
\end{aligned}
$$




$$
\begin{aligned}
& \times\left(\iint \widetilde{S}(\tau, v) A_{q l}\left(\tau_{2}+n T, v_{2}+m F\right)\right. \\
& \left.\left.\cdot e^{-j \pi\left(\tau_{2}+n T\right)\left(v_{2}-m F\right)} d \tau_{2} d \nu_{2}\right)\right\}
\end{aligned}
$$

with $A_{p k}(\tau, \nu)$ being the Hermite cross-ambiguity function. The latter function is defined as

$$
\begin{aligned}
A_{p k}(\tau, v) & =\int h_{p}\left(t-\frac{\tau}{2}\right) h_{k}\left(t+\frac{\tau}{2}\right) e^{-j 2 \pi v t} d t \\
& = \begin{cases}(-\sqrt{\pi})^{p-k} \sqrt{\frac{k !}{p !}} e^{(-\pi / 2)\left(\tau^{2}+\nu^{2}\right)}(\tau+j \nu)^{p-k} L_{k}^{p-k}\left(\pi\left(\tau^{2}+v^{2}\right)\right), & \text { if } p \geq k, \\
(\sqrt{\pi})^{k-p} \sqrt{\frac{p !}{k !}} e^{(-\pi / 2)\left(\tau^{2}+v^{2}\right)}(\tau-j \nu)^{k-p} L_{p}^{k-p}\left(\pi\left(\tau^{2}+v^{2}\right)\right), & \text { else, }\end{cases}
\end{aligned}
$$

where $L_{n}^{a}(\cdot)$ is the Laguerre polynomial [20].

Introducing the vectors $\boldsymbol{\alpha}=\left(\alpha_{0}, \ldots, \alpha_{K}\right)^{T}$ and $\boldsymbol{\beta}=$ $\left(\beta_{0}, \ldots, \beta_{K}\right)^{T}$, we can express the SINR, in matrix form, either as

$$
\operatorname{SINR}=\frac{\boldsymbol{\beta}^{T} \mathbf{A}_{\boldsymbol{\alpha}} \boldsymbol{\beta}}{\boldsymbol{\beta}^{T} \mathbf{B}_{\boldsymbol{\alpha}} \boldsymbol{\beta}}
$$

where

$$
\begin{aligned}
& \left(\mathbf{A}_{\boldsymbol{\alpha}}\right)_{k l}=\sum_{p, q=0}^{K} \alpha_{p} \alpha_{q} M_{k l p q}^{(00)}, \\
& \left(\mathbf{B}_{\boldsymbol{\alpha}}\right)_{k l}=\sum_{p, q=0}^{K} \alpha_{p} \alpha_{q} \sum_{\substack{m n \\
(m, n) \neq(0,0)}} M_{k l p q}^{(m n)}+\left(1+\delta_{i}\right) \frac{N_{0}}{\bar{E}}\|\boldsymbol{\alpha}\|^{2},
\end{aligned}
$$

or as

$$
\operatorname{SINR}=\frac{\boldsymbol{\alpha}^{T} \mathbf{A}_{\boldsymbol{\beta}} \boldsymbol{\alpha}}{\boldsymbol{\alpha}^{T} \mathbf{B}_{\boldsymbol{\beta}} \boldsymbol{\alpha}}
$$

where

$$
\begin{aligned}
& \left(\mathbf{A}_{\boldsymbol{\beta}}\right)_{p q}=\sum_{k, l=0}^{K} \beta_{k} \beta_{l} M_{k l p q}^{(00)}, \\
& \left(\mathbf{B}_{\boldsymbol{\beta}}\right)_{p q}=\sum_{k, l=0}^{K} \beta_{k} \beta_{l} \sum_{\substack{m n \\
(m, n) \neq(0,0)}} M_{k l p q}^{(m n)}+\left(1+\delta_{i}\right) \frac{N_{0}}{\bar{E}}\|\boldsymbol{\beta}\|^{2} .
\end{aligned}
$$

\section{POPS Optimization Algorithm}

The optimization problem at hand is defined as

$$
\left(\boldsymbol{\alpha}_{\mathrm{opt}}, \boldsymbol{\beta}_{\mathrm{opt}}\right)=\arg \max _{(\boldsymbol{\alpha}, \boldsymbol{\beta})} \operatorname{SINR}
$$

Given the special forms of the SINR expressions in (35) and (37), it is easy to see that the optimization problem is equivalent to a maximization of a generalized Rayleigh quotient. For finite, yet practical, values of the SNR, $N_{0} / \bar{E}$ is nonnull, and $\left(N_{0} / \bar{E}\right)\|\boldsymbol{\alpha}\|^{2}$ and $\left(N_{0} / \bar{E}\right)\|\boldsymbol{\beta}\|^{2}$ are trivial quadratic forms which, when added to the positive Hermitian quadratic forms in the expressions of $\mathbf{B}_{\boldsymbol{\alpha}}$ and $\mathbf{B}_{\boldsymbol{\beta}}$, guarantee their invertibility and their relative well-conditioning.

The POPS approach, which is proposed to optimize the $\mathrm{Tx} / \mathrm{Rx}$ waveforms, is detailed in Algorithm 1, where $N$ and $M$ are such that $(2 N+1)$ is the number of FBMC symbols and $(2 M+1)$ is the number of subcarriers. The main steps of the optimization algorithm are the following:

Step 1. We compute the matrix entries $M_{k l p q}^{(m n)}$ in (33), where $k, l, p, q \in \llbracket 0, K \rrbracket, m \in \llbracket-M, M \rrbracket$ and $n \in \llbracket-N, N \rrbracket$.

Step 2. In the initialization step of Algorithm 1, we start by an arbitrary nonnull vector $\boldsymbol{\alpha}^{(0)}$, typically $(1,0, \ldots, 0)^{T}$, meaning that the starting waveform is the most localized Gaussian function.

Step 3. For iteration (i), we compute $\boldsymbol{\beta}^{(i)}$ as the eigenvector of $\boldsymbol{\Omega}_{\boldsymbol{\alpha}}^{(i)}$ with maximum eigenvalue.

Step 4. Given $\boldsymbol{\beta}^{(i)}$, we determine $\boldsymbol{\alpha}^{(i+1)}$ as the eigenvector of $\boldsymbol{\Omega}_{\boldsymbol{\beta}}^{(i)}$ with maximum eigenvalue.

Step 5. We proceed to the next iteration $(i+1)$.

Step 6. We stop the iterations when we obtain a negligible increase of SINR.

Before proceeding, it is deemed useful to emphasize, for those who are familiar with the Lloyd-Max algorithm [21], which is used in scalar or vector quantizer optimization, its strong similarity with the POPS algorithm. Indeed, while the Lloyd-Max algorithm alternates between an optimization of the quantization regions (or intervals in the scalar case) and their representatives, the POPS algorithm alternates between an optimization of the Tx waveform and the Rx waveform.

\section{Simulation Results}

In this section, we evaluate the performance of the POPS algorithm in FBMC/OQAM systems and conduct a comparison with FBMC/QAM systems, in terms of SINR. To this 


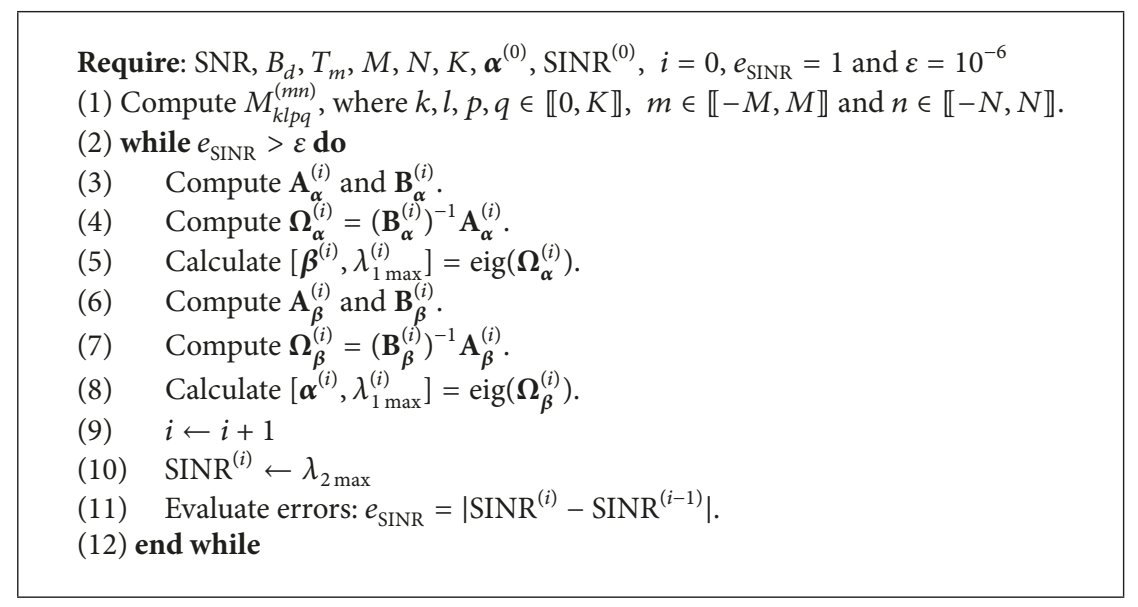

Algorithm 1: POPS algorithm.

end, we first specify the scattering function of the channel needed in the calculation of the SINR expression in (32). Actually, an infinite number of scattering function models, to which the transmitted signal can be exposed, occur in practice. While the POPS based optimization of the Tx and $\mathrm{Rx}$ waveforms is applicable for whatever WSSUS channel, we limit ourselves, in the absence of information on the actual scattering function, to the maxentropic [22], flat, and normalized scattering function

$$
\widetilde{S}(\tau, \nu)= \begin{cases}\frac{1}{B_{d} T_{m}}, & \text { if }|\tau| \leq \frac{T_{m}}{2},|\nu| \leq \frac{B_{d}}{2} \\ 0, & \text { else, }\end{cases}
$$

where $B_{d}$ and $T_{m}$ are the Doppler spread and the delay spread, respectively.

In general, all Hermite functions whose indices are between 0 and $K$ are important in waveforms representation. However, for the case of the flat rectangular scattering function considered in (40), the adopted system model can, without any loss of generality, be casted to an equivalent system model with a scattering function of square form as well as a time-frequency lattice of square cells, by means of a balanced distribution of delay and Doppler spreads in time and frequency, respectively (i.e., $B_{d} / F=T_{m} / T$ ), followed by a scaling of the time axis specified in detail in Appendix 1. The symmetry of time and frequency axes acquired by this scaling allows us to restrict the optimization process to waveforms with the same shape in time and frequency, that is, with identical Fourier transforms, up to multiplicative factors of unit modulus. Thus, in the expansion of the waveforms to be optimized, we can restrict ourselves to Hermite functions with indices in $\llbracket 0, K \rrbracket$ of the form $4 l+r$, where $l$ is a nonnegative integer and $r \in \llbracket 0,3 \rrbracket$. Since the choice $r=0$ guarantees the best concentration and localization in time and frequency, we only keep, for our expansion, Hermite functions with indices between 0 and $K$ which are integer multiples of 4 , offering a reduction of POPS optimization complexity by around a factor of 4 . As a consequence, in the evaluation of the SINR, the total number of entries $M_{k l p q}$ to be computed is approximately reduced by a factor of $4^{4}=256$. Moreover, given the similar shape in time and frequency of the waveforms within the restricted expansion space, we can set $N=M$ in all considered simulations.

Generally, the double integrals in (33) do not possess closed-form expressions for arbitrary expressions of the scattering function. As a consequence, for their evaluation, we resort to a straightforward, yet time and resource consuming, Riemannian numerical integration. To efficiently approximate these double integrals, we increase the number of subdivisions for each square $\left[-B_{d} / 2, B_{d} / 2\right] \times\left[-T_{m} / 2, T_{m} / 2\right]$ until reaching numerical stability. Then, for each number of Hermite functions, we increase the number of FBMC symbols, $N$, which is equal to the number of subcarriers, $M$, until having a stable SINR value. Therefore, we calculate the SINR for each optimal couple $(K, N)$, for different values of $B_{d} T_{m}$ and SNR.

To justify the range of practical values of $B_{d} T_{m}$, to be adopted in all carried simulations, we emphasize that each transmitted signal experiences time and frequency spreads that are the compound effects of natural and artificial phenomena. Frequency spread is incurred by the joint effects of the Doppler spread, caused by the channel, and the residual frequency synchronization errors, due to the receiver. Time spread is the result of the delay spread due to the channel and the residual time synchronization errors induced by the receiver, altogether, with the time misalignment caused by the multiple access nature of the transmission system. Based on the values of $T_{m}$ given in [23], we provide in Table 1 some typical values of $B_{d} T_{m}$ for practical channels when the carrier frequency, $f_{C}$, is set to $2 \mathrm{GHz}$. Note that channel A and channel $\mathrm{B}$ models are, respectively, the low and median delay spread channel models that typically occur in practice. It is important to stress that the obtained values of $B_{d} T_{m}$ in Table 1 , which are in the range $\left[10^{-6}, 10^{-3}\right]$, must be casted to the higher range, $\left[10^{-3}, 10^{-2}\right]$, when artificial imperfections are added up.

In practice, due to complexity and memory consumption considerations, we cannot have a pair of optimized waveforms for each value of $B_{d} T_{m}$. As a consequence, we choose, in 
TABLE 1: Typical values of $B_{d} T_{m}$, due to natural phenomena, for practical channels [23].

\begin{tabular}{|c|c|c|c|c|c|c|}
\hline & \multicolumn{2}{|c|}{ Indoor office } & \multicolumn{2}{|c|}{ Outdoor-to-indoor and pedestrian } & \multicolumn{2}{|c|}{ Vehicular } \\
\hline & Channel A & Channel B & Channel A & Channel B & Channel A & Channel B \\
\hline Speed $(\mathrm{km} / \mathrm{h})$ & & & 3 & & & \\
\hline$B_{d}(\mathrm{~Hz})$ & & & 1.11 & & & \\
\hline$T_{m}(\mathrm{~ns})$ & 35 & 100 & 45 & 750 & 370 & 4000 \\
\hline$B_{d} T_{m}$ & $0.389 \cdot 10^{-6}$ & $1.11 \cdot 10^{-6}$ & $0.5 \cdot 10^{-6}$ & $8.33 \cdot 10^{-6}$ & $1.64 \cdot 10^{-4}$ & $1.77 \cdot 10^{-3}$ \\
\hline
\end{tabular}

TABLE 2: Intervals of values of $B_{d} T_{m}$ and their representatives.

\begin{tabular}{lc}
\hline Interval & Representative \\
\hline $10^{-2.5}<B_{d} T_{m}$ & $10^{-2}$ \\
$10^{-3.5}<B_{d} T_{m} \leq 10^{-2.5}$ & $10^{-3}$ \\
$10^{-4.5}<B_{d} T_{m} \leq 10^{-3.5}$ & $10^{-4}$ \\
$10^{-5.5}<B_{d} T_{m} \leq 10^{-4.5}$ & $10^{-5}$ \\
$B_{d} T_{m} \leq 10^{-5.5}$ & $10^{-6}$ \\
\hline
\end{tabular}

offline mode, some meaningful representative values of $B_{d} T_{m}$, for which we optimize the $\mathrm{Tx} / \mathrm{Rx}$ waveforms, as shown in Table 2. The obtained pairs of waveforms for these retained values of $B_{d} T_{m}$ form a dictionary that can be used in practice for all $B_{d} T_{m}$ values around the value of their representative.

7.1. FBMC/OQAM Systems Performance. For FBMC/OQAM systems, we focus on the case of a critical lattice density, with $F T=1 / 2$. To initialize the POPS algorithm, we evaluate the SINR, for each value of $K$, for $\boldsymbol{\alpha}^{(0)}=(1,0, \ldots, 0)^{T}$, which means that the initializing waveform, $\varphi^{(0)}$, is the most localized Gaussian function. We note that the SINR stabilizes at $K=16$. To be sure that we have obtained the optimal SINR and that we have not been trapped to a local maximum of the SINR, we resort to systematic initializations, arising from a thinly quantized browsing of the space of allowed solutions. More specifically, we scan the initializations space $\mathbb{R}^{K / 4+1}=$ $\mathbb{R}^{5}$ (which corresponds to the dimensions of $\boldsymbol{\alpha}^{(0)}$ for $K=16$ ), as follows. We start by thinly quantifying this space. Then we choose the elements of $\boldsymbol{\alpha}^{(0)}$, using the spherical coordinates of dimension $K / 4+1$. In this way, we are able to browse all possible quantized initializations.

In Figure 1, we compare the optimal couple of $\mathrm{Tx} / \mathrm{Rx}$ waveforms $\left(\varphi_{\text {opt }}, \psi_{\text {opt }}\right)$, which maximizes the SINR, with the IOTA waveform, first introduced in [24]. We note that the optimal Tx/Rx waveforms, resulting from Algorithm 1, decrease faster than the IOTA waveform, since we use a finite number of Hermite functions that are known to decrease exponentially (in $e^{-\pi t^{2}}$ to be more specific), while the IOTA function witnesses a decrease in $e^{-|t|}$. Therefore, they are more localized in time and can be truncated to a shorter time duration when it comes to practical hardware realizations. Indeed, with a reduced truncation duration, we are able to use fewer samples to realize any signal processing at the Tx or the $\mathrm{Rx}$ involving any filtering with the Tx and Rx waveforms. Therefore, it will be easier to realize in practice.

Figure 2 presents the evolution of the SINR as a function of $K$, for $\mathrm{SNR}=30 \mathrm{~dB}$. Numerical results in this figure show

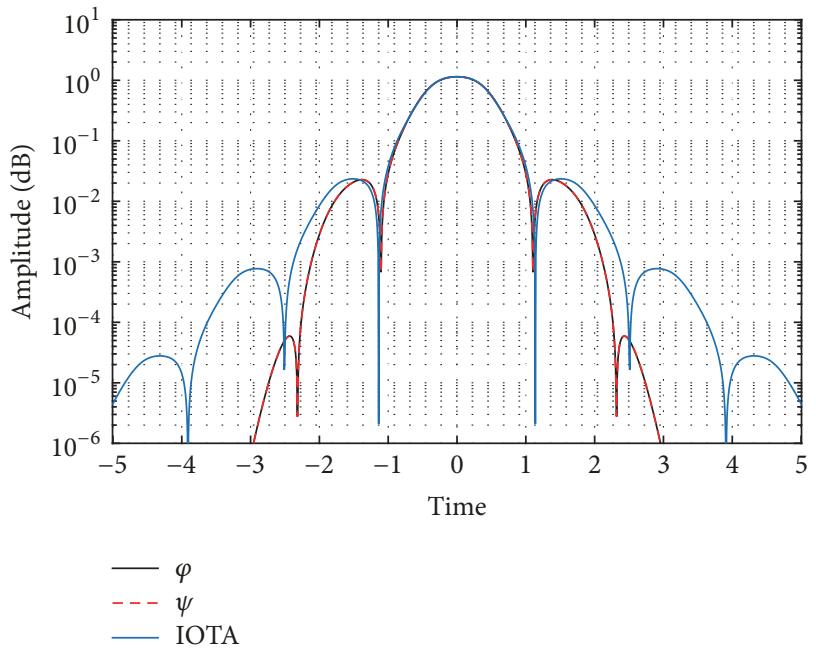

FIgURE 1: Comparison of the POPS waveforms for $B_{d} T_{m}=10^{-4}$ with the IOTA waveform, in FBMC/OQAM systems.

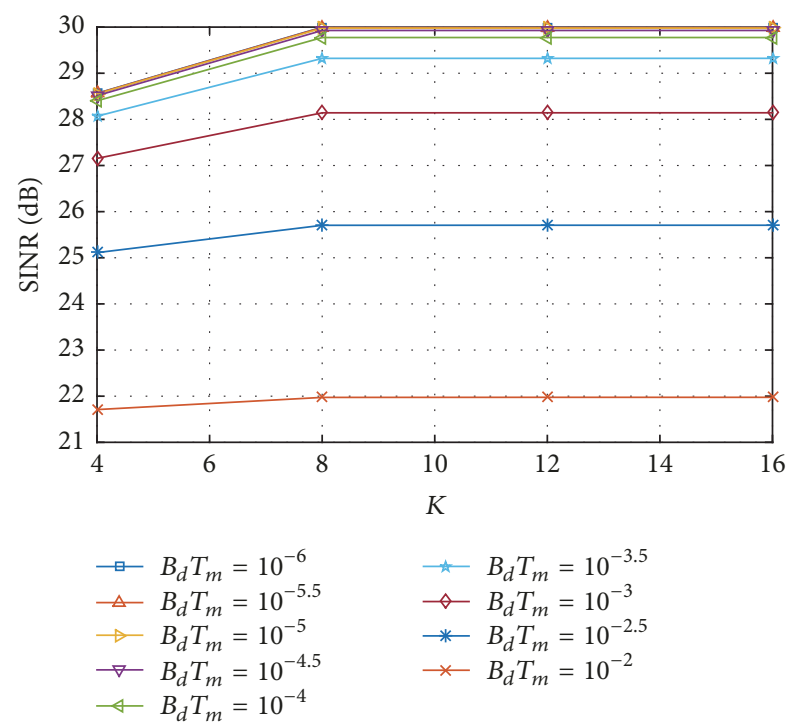

FIgURE 2: Optimal SINR as a function of $K$, for FBMC/OQAM systems, for $\mathrm{SNR}=30 \mathrm{~dB}$.

that, by decreasing the time-frequency dispersions, the SINR increases until converging to the SNR for $B_{d} T_{m}=10^{-6}$. We note also that the SINR enhances with the number of Hermite functions and becomes stable at $K=16$. 


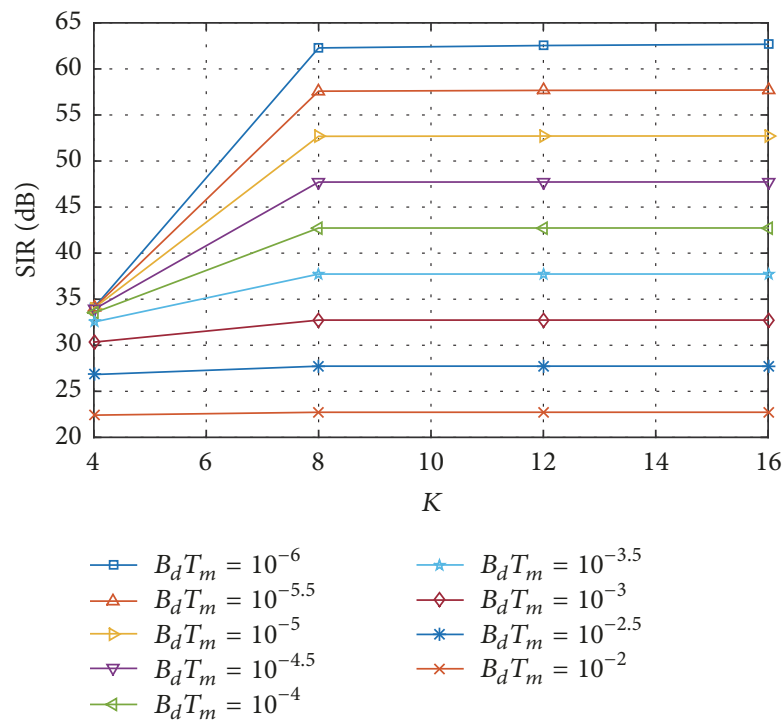

FIgURE 3: Optimal SIR as a function of $K$, for FBMC/OQAM systems.

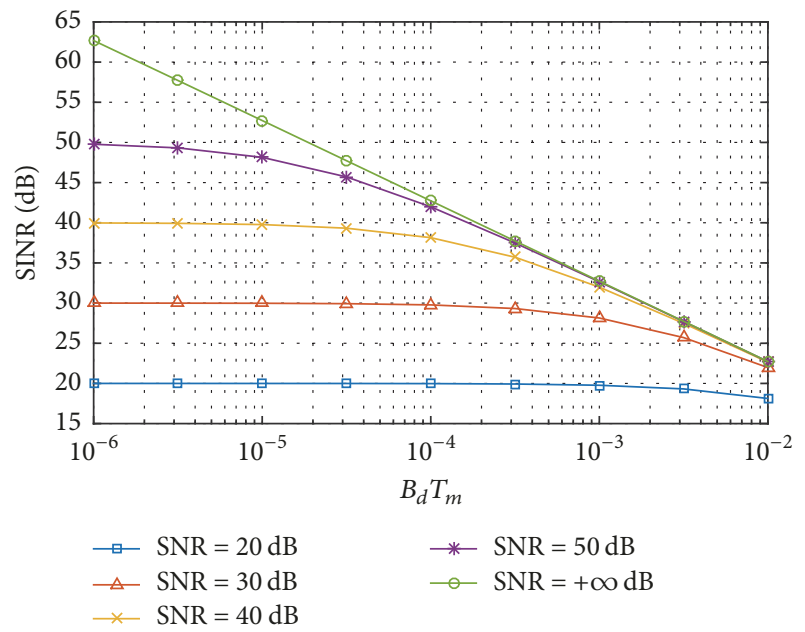

FIgUre 4: Optimal SINR as a function of $B_{d} T_{m}$, for FBMC/OQAM systems, for $\mathrm{SNR}=20,30,40,50$ and $+\infty \mathrm{dB}$.

Figure 3 illustrates the evolution of the SIR as a function of $K$. Note that the SIR is equal to the SINR when the thermal noise is perfectly null, that is, when SNR $=+\infty \mathrm{dB}$. It enables the measurement of the transmission chain quality when it operates under good conditions with a negligible noise compared to ISI. As in Figure 2, we notice that the SIR increases with $K$ and stabilizes at $K=16$. Besides, the SIR enhances following a decrease in $B_{d} T_{m}$, thanks to a reduction in channel dispersion severity, which is accompanied with an alleviation of the overlap between the $\varphi_{m n}$ in the timefrequency plane and therefore by a decrease in interference.

Figure 4 presents the evolution of the SINR as a function of $B_{d} T_{m}$, for $\mathrm{SNR}=20,30,40,50$ and $+\infty \mathrm{dB}$. We notice that, for each SNR value, for low $B_{d} T_{m}$, the interference becomes null; thus, the SINR $=$ SNR. However, for high $B_{d} T_{m}$, the interference becomes dominant; therefore, the SINR $<$ SNR.

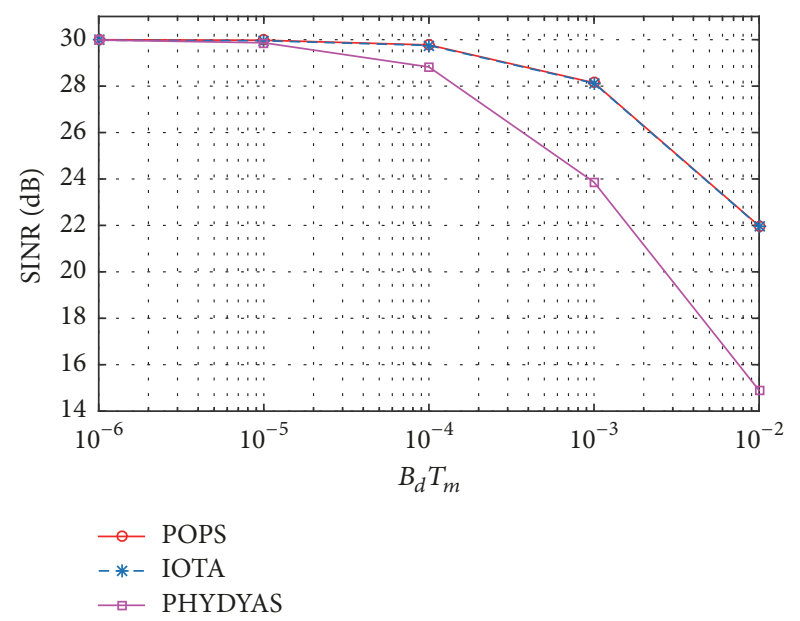

FIgURE 5: SINR of POPS versus IOTA and PHYDYAS as a function of $B_{d} T_{m}$, for FBMC/OQAM systems and SNR $=30 \mathrm{~dB}$.

Furthermore, even for high SNR, the SINR degrades rapidly, even for low dispersions, while, for small SNR values, even for high dispersions, the SINR remains close to the SNR, because the interference always remains lower than the noise.

Figure 5 shows the evolution of the SINR as a function of $B_{d} T_{m}$, for SNR $=30 \mathrm{~dB}$. In this figure, we compare the POPS optimal SINR, given by $K=16$, to the PHYDYAS SINR, while using an overlapping factor of 4 [25], and to the IOTA SINR. The obtained results demonstrate that the POPS algorithm improves the SINR with a gain of $7.09 \mathrm{~dB}$ for high dispersions $\left(B_{d} T_{m}=10^{-2}\right)$, compared to PHYDYAS. It should be noted that PHYDYAS is a prototype filter, recently defined in PHYDYAS Project [25], and used in FBMC/OQAM systems in 5GNOW Project [26]. Numerical results also show that the POPS optimal SINR is slightly better than the IOTA SINR with a rough gain of $0.2 \mathrm{~dB}$, which proves that the IOTA waveform has the nice property of offering a good performance in terms of SINR. Yet, as shown in Figure 6, the optimal POPS $\mathrm{Tx} / \mathrm{Rx}$ waveforms are more localized in frequency and therefore offer a dramatic reduction in out-ofband $(\mathrm{OOB})$ emissions with respect to the IOTA waveform.

7.2. FBMC/OQAM Systems versus FBMC/QAM Systems. Since we have used lattice densities close or equal to the critical density in FBMC/QAM systems, the convergence of the POPS algorithm becomes more difficult. Thus, we will use a high number of Hermite functions. Therefore, we cannot evaluate all possible combinations of the components of $\boldsymbol{\alpha}$, for each value of $K$. Hence, we will use a high number of random combinations to obtain the optimal $\mathrm{Tx} / \mathrm{Rx}$ waveforms coefficient couple $\left(\boldsymbol{\alpha}_{\text {opt }}, \boldsymbol{\beta}_{\text {opt }}\right)$, which maximizes the SINR.

Figures 7, 8, and 9 illustrate the evolution of the optimal SINR as a function of $K$, for SNR $=30 \mathrm{~dB}$ and different values of $B_{d} T_{m}$. We note that the higher $F T$ is, the faster the stabilization of the SINR is. It is better to say that the lower the lattice density is, the better the convergence of the SINR to the SNR, for low dispersions, is. We note also that the SINR 


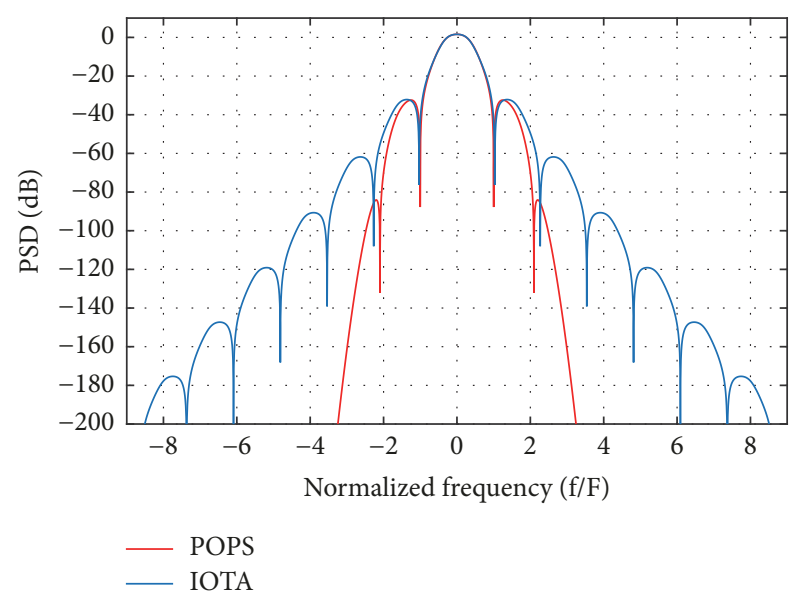

Figure 6: PSD of POPS versus IOTA, for FBMC/OQAM systems.

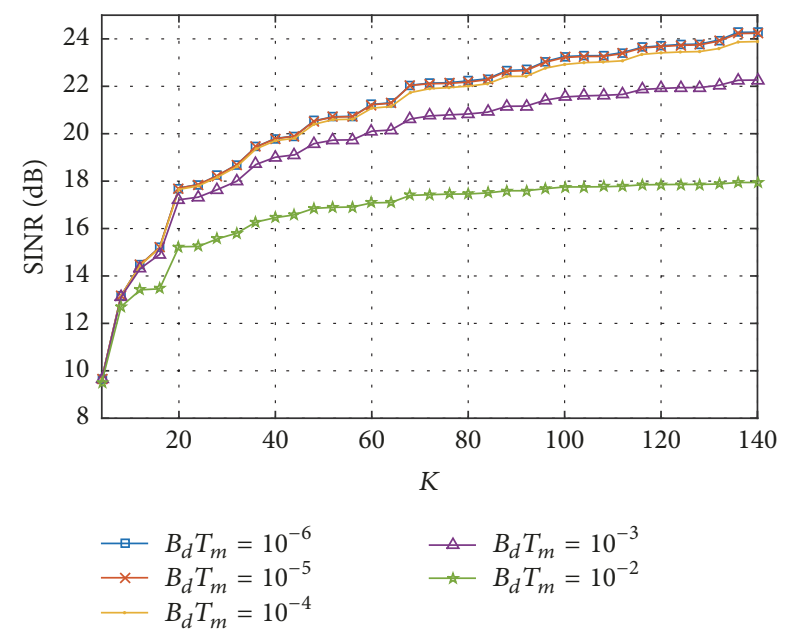

FIgure 7: Optimal SINR as a function of $K$, for FBMC/QAM systems, for $\mathrm{SNR}=30 \mathrm{~dB}$ and $F T=1$.

becomes stable for high values of $K$, in FBMC/QAM systems, since the lattice densities are close to 1 . Therefore, the optimal $\mathrm{Tx} / \mathrm{Rx}$ waveforms will not be well localized in time and frequency. Unlike FBMC/QAM systems, for FBMC/OQAM systems, as shown in Figure 2, the SINR converges at $K=16$. Hence, the obtained Tx/Rx waveforms will be well localized in time and frequency. In Figure 7, for critical density equal to 1 , the SINR of FBMC/QAM systems does not reach the SNR even for $K=140$ and $B_{d} T_{m}=10^{-6}$. By moving away gradually from the critical density, and for very low dispersions, the SINR reaches the SNR for $K=140$ and $F T=1+1 / 32$, as shown in Figure 8, and for $K=100$ and $F T=1+1 / 16$, as revealed in Figure 9 .

Figure 10 is obtained by selecting the optimal value of the SINR, for each value of $B_{d} T_{m}$, in Figures $2,7,8$, and 9. For $B_{d} T_{m}=10^{-2}$ and a critical density $(\Delta=2$ for FBMC/OQAM and $\Delta=1$ for FBMC/QAM), FBMC/OQAM outperforms FBMC/QAM by $4.04 \mathrm{~dB}$. On the other hand, for $\Delta=1 /(1+1 / 32) \simeq 0.97$, the difference between the two systems falls to $0.29 \mathrm{~dB}$. Finally, for $\Delta=1 /(1+1 / 16) \simeq 0.94$, FBMC/QAM outperforms FBMC/OQAM by $1.03 \mathrm{~dB}$. We

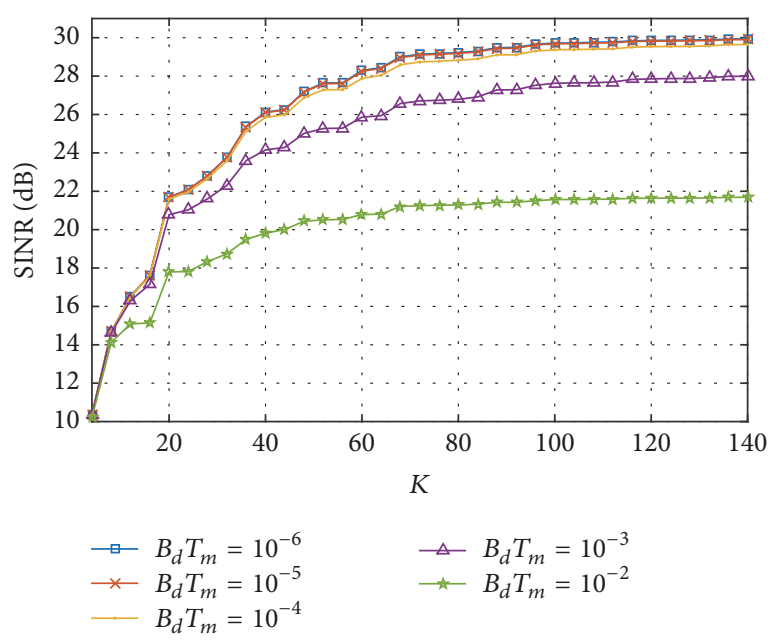

FIGURE 8: Optimal SINR as a function of $K$, for FBMC/QAM systems, for $\mathrm{SNR}=30 \mathrm{~dB}$ and $F T=1+1 / 32$.

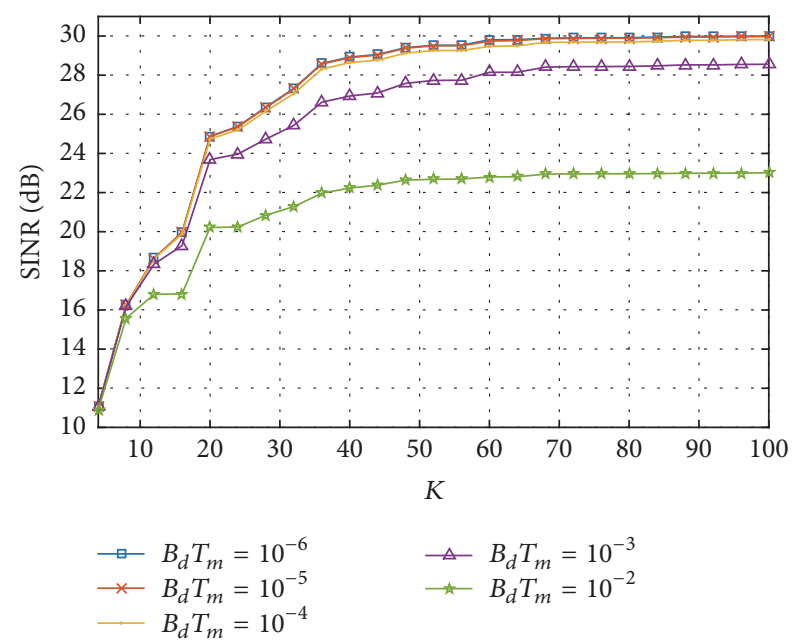

FIgURE 9: Optimal SINR as a function of $K$, for FBMC/QAM systems, for $\mathrm{SNR}=30 \mathrm{~dB}$ and $F T=1+1 / 16$.

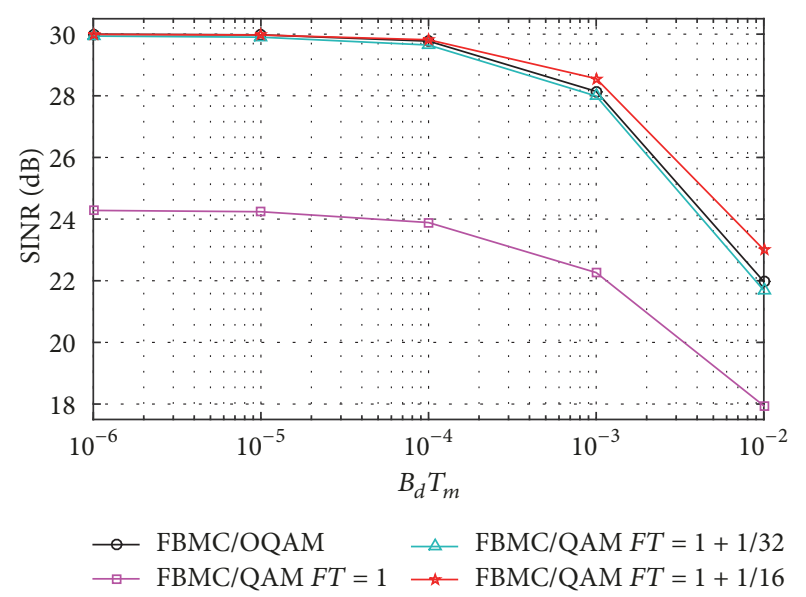

FIgURE 10: Optimal SINR of FBMC/OQAM systems versus FBMC/QAM systems, as a function of $B_{d} T_{m}$, for $\mathrm{SNR}=30 \mathrm{~dB}$. 
learn from these considerations that FBMC/QAM is capable of offering a comparable performance to FBMC/OQAM, in terms of SINR, at the price of a slight decrease, in the order of $1 / 32$, of the time-frequency lattice density. This reduction, while being judged insignificant, has the great merit of drastically simplifying the complexity of the Tx and the $\mathrm{Rx}$, especially in the presence of MIMO systems. We note that, for $B_{d} T_{m}=10^{-6}$, all curves of SINR converge to the SNR except for $F T=1$ in FBMC/QAM systems, because the POPS algorithm execution requires too much time for $K>140$.

\section{Conclusion}

In this paper, we investigated an optimal waveform design for multi-carrier transmissions over rapidly time-varying and strongly delay spread channels, in FBMC/OQAM systems. To this end, we derived the SINR analytical expression, which is one of the most important and challenging results of this study. Then, we extended the POPS algorithm, which was first applied to FBMC/QAM systems, to FBMC/OQAM systems, in order to design optimal $\mathrm{Tx}$ and $\mathrm{Rx}$ waveforms, providing a high reduction in ICI and ISI, and guaranteeing an effective maximization of the SINR. Besides, we have verified, theoretically, the excellent performance of FBMC/OQAM systems with respect to FBMC/QAM systems for critical densities. We also discovered that a slight decrease in time-frequency lattice density, or equivalently in spectrum efficiency, allows FBMC/QAM systems to outperform FBMC/OQAM systems. However, in terms of waveforms localization in time and frequency, FBMC/OQAM remains significantly better than FBMC/QAM. Despite the superiority of FBMC/OQAM in terms of SINR and waveform localization at critical density, we believe that FBMC/QAM should be recommended in practice. Indeed, the implementation complexity of FBMC/OQAM increases excessively, when we move from SISO to more widespread MIMO systems, and does not justify the slight gain of around 5\% procured in spectral efficiency, compared to FBMC/QAM. A possible challenging research axis of the presented work consists in extending this comparison to the case of discrete time, hexagonal timefrequency lattices, and multipulse systems. Although our research team has already worked on multipulse systems in [27], we believe that this concept is topical and continues to present new perspectives through the adoption of the POPS paradigm for both FBMC/OQAM and FBMC/QAM systems.

\section{Appendix}

\section{A. Derivation of the Useful and Interference Powers in FBMC/OQAM Systems}

In this appendix, we provide the details of the derivation of the useful and interference powers in FBMC/OQAM systems, that is, $P_{U}$ and $P_{I}$. First, we set

$$
X=C(0 ; 0)=\sum_{l=0}^{L-1} c_{l}
$$

$$
\begin{aligned}
& Y_{m n}=\left\langle\psi_{00}, \widetilde{\varphi}_{m n}\right\rangle, \\
& P_{m n}=\frac{E}{2\|\varphi\|^{2}} \mathbb{E}\left[\left(\Re\left\{e^{-j \chi_{00}}\left\langle\psi_{00}, \widetilde{\varphi}_{m n}\right\rangle\right\}\right)^{2}\right] .
\end{aligned}
$$

Since the compensation factor is given by $e^{j \chi_{00}}=C(0 ; 0) / \mid C(0$; $0)|=X /| X \mid(\operatorname{see}(9))$, we will have $e^{-j \chi_{00}}\left\langle\psi_{00}, \widetilde{\varphi}_{m n}\right\rangle=X^{*} Y_{m n} /$ $|X|$. Therefore, we can write

$$
P_{m n}=\frac{E}{2\|\varphi\|^{2}} \mathbb{E}\left[\left(\mathfrak{R}\left\{\frac{X^{*}}{|X|} Y_{m n}\right\}\right)^{2}\right] .
$$

On the other hand, $Y_{m n}$ can be expressed as

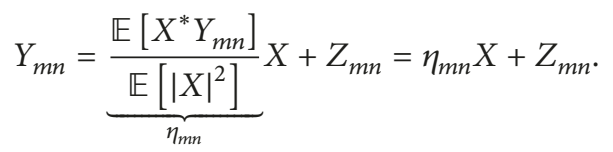

Thus, we will have $Z_{m n}=Y_{m n}-\eta_{m n} X$. Consequently, the intercorrelation between $X$ and $Z_{m n}$ is given by $\mathbb{E}\left[X^{*} Z_{m n}\right]=$ $\mathbb{E}\left[X^{*} Y_{m n}\right]-\eta_{m n} \mathbb{E}\left[|X|^{2}\right]=0$. Therefore, $X$ and $Z_{m n}$ are two independent random complex Gaussian variables. Hence, we can write

$$
\begin{aligned}
\frac{X^{*}}{|X|} Y_{m n} & =\frac{X^{*}}{|X|}\left(\eta_{m n} X+Z_{m n}\right)=\frac{\eta_{m n}|X|^{2}}{|X|}+\underbrace{\frac{X^{*}}{|X|} Z_{m n}}_{V_{m n}} \\
& =\eta_{m n}|X|+V_{m n},
\end{aligned}
$$

where $V_{m n}$ is a random complex Gaussian variable, which is independent of $X$ and has the same variance of $Z_{m n}$. Accordingly, we obtain

$$
\begin{aligned}
\Re\left\{\frac{X^{*}}{|X|} Y_{m n}\right\} & =\Re\left\{\eta_{m n}|X|+V_{m n}\right\} \\
& =\underbrace{\Re\left\{\eta_{m n}\right\}}_{\varepsilon_{m n}}|X|+\underbrace{\mathfrak{R}\left\{V_{m n}\right\}}_{W_{m n}} \\
& =\varepsilon_{m n}|X|+W_{m n},
\end{aligned}
$$

where $\varepsilon_{m n}=\Re\left\{\eta_{m n}\right\}=\Re\left\{\mathbb{E}\left[X^{*} Y_{m n}\right]\right\} / \mathbb{E}\left[|X|^{2}\right]$ and $W_{m n}$ is a random real Gaussian variable, which is independent of $|X|$ and has a zero mean and the half variance of $Z_{m n}$, that is, $\mathbb{E}\left[\left|W_{m n}\right|^{2}\right]=(1 / 2) \mathbb{E}\left[\left|V_{m n}\right|^{2}\right]=(1 / 2) \mathbb{E}\left[\left|Z_{m n}\right|^{2}\right]$. As a consequence, we can write

$$
\begin{aligned}
\mathbb{E}\left[\left(\Re\left\{\frac{X^{*}}{|X|} Y_{m n}\right\}\right)^{2}\right]=\varepsilon_{m n}^{2} \mathbb{E}\left[|X|^{2}\right]+\mathbb{E}\left[\left|W_{m n}\right|^{2}\right] \\
=\frac{\left(\Re\left\{\mathbb{E}\left[X^{*} Y_{m n}\right]\right\}\right)^{2}}{\left(\mathbb{E}\left[|X|^{2}\right]\right)^{2}} \mathbb{E}\left[|X|^{2}\right]+\frac{1}{2} \mathbb{E}\left[\left|Z_{m n}\right|^{2}\right] \\
=\frac{\left(\Re\left\{\mathbb{E}\left[X^{*} Y_{m n}\right]\right\}\right)^{2}}{\mathbb{E}\left[|X|^{2}\right]}+\frac{1}{2} \mathbb{E}\left[\left|Z_{m n}\right|^{2}\right] .
\end{aligned}
$$


Since we have $Z_{m n}=Y_{m n}-\eta_{m n} X$, the variance of $Z_{m n}$ can be expressed as

$$
\begin{aligned}
\mathbb{E}\left[\left|Z_{m n}\right|^{2}\right]= & \mathbb{E}\left[\left|Y_{m n}\right|^{2}\right]+\left|\eta_{m n}\right|^{2} \mathbb{E}\left[|X|^{2}\right] \\
& -\mathbb{E}\left[\beta_{m n}^{*} X^{*} Y_{m n}\right]-\mathbb{E}\left[Y_{m n}^{*} \eta_{m n} X\right] \\
= & \mathbb{E}\left[\left|Y_{m n}\right|^{2}\right]+\frac{\left|\mathbb{E}\left[X^{*} Y_{m n}\right]\right|^{2}}{\left(\mathbb{E}\left[|X|^{2}\right]\right)^{2}} \mathbb{E}\left[|X|^{2}\right] \\
& -\beta_{m n}^{*} \mathbb{E}\left[X^{*} Y_{m n}\right]-\eta_{m n} \mathbb{E}\left[X Y_{m n}^{*}\right] \\
= & \mathbb{E}\left[\left|Y_{m n}\right|^{2}\right]+\frac{\left|\mathbb{E}\left[X^{*} Y_{m n}\right]\right|^{2}}{\mathbb{E}\left[|X|^{2}\right]} \\
& -\frac{\left(\mathbb{E}\left[X^{*} Y_{m n}\right]\right)^{*}}{\mathbb{E}\left[|X|^{2}\right]}\left[X^{*} Y_{m n}\right] \\
& -\frac{\mathbb{E}\left[X^{*} Y_{m n}\right]}{\mathbb{E}\left[|X|^{2}\right]} \mathbb{E}\left[X Y_{m n}^{*}\right] \\
= & \mathbb{E}\left[\left|Y_{m n}\right|^{2}\right]-\frac{\left|\mathbb{E}\left[X^{*} Y_{m n}\right]\right|^{2}}{\mathbb{E}\left[|X|^{2}\right]} .
\end{aligned}
$$

Injecting (A.7) in (A.6), we can write

$$
\begin{aligned}
\mathbb{E}\left[\left(\mathfrak{R}\left\{\frac{X^{*}}{|X|} Y_{m n}\right\}\right)^{2}\right] \\
=\frac{\left(\mathfrak{R}\left\{\mathbb{E}\left[X^{*} Y_{m n}\right]\right\}\right)^{2}}{\mathbb{E}\left[|X|^{2}\right]}+\frac{1}{2} \mathbb{E}\left[\left|Y_{m n}\right|^{2}\right] \\
-\frac{1}{2} \frac{\left|\mathbb{E}\left[X^{*} Y_{m n}\right]\right|^{2}}{\mathbb{E}\left[|X|^{2}\right]} .
\end{aligned}
$$

Using the following relation $\left(\mathfrak{R}\left\{\mathbb{E}\left[X^{*} Y_{m n}\right]\right\}\right)^{2}=(1 /$ 2) $\left(\Re\left\{\left(\mathbb{E}\left[X^{*} Y_{m n}\right]\right)^{2}\right\}+\left|\mathbb{E}\left[X^{*} Y_{m n}\right]\right|^{2}\right)$, the expression in (A.8) can be written as

$$
\begin{aligned}
& \mathbb{E}\left[\left(\Re\left\{\frac{X^{*}}{|X|} \mathrm{Y}_{m n}\right\}\right)^{2}\right] \\
& =\frac{1}{2}\left(\frac{\Re\left\{\left(\mathbb{E}\left[X^{*} Y_{m n}\right]\right)^{2}\right\}}{\mathbb{E}\left[|X|^{2}\right]}+\mathbb{E}\left[\left|Y_{m n}\right|^{2}\right]\right) .
\end{aligned}
$$

As a result, $P_{m n}$ will be given by

$$
P_{m n}=\frac{E}{4\|\varphi\|^{2}}\left(\frac{\Re\left\{\left(\mathbb{E}\left[X^{*} Y_{m n}\right]\right)^{2}\right\}}{\mathbb{E}\left[|X|^{2}\right]}+\mathbb{E}\left[\left|Y_{m n}\right|^{2}\right]\right) .
$$

As $Y_{m n}$ is equal to

$$
\begin{aligned}
Y_{m n} & =\left\langle\psi_{00}, \tilde{\varphi}_{m n}\right\rangle=\int \psi_{00}^{*}(t) \tilde{\varphi}_{m n}(t) d t \\
& =\int \psi_{00}^{*}(t)\left(\int c(\tau ; t) \varphi_{m n}(t-\tau) d \tau\right) d t \\
& =\int \psi_{00}^{*}(t) \sum_{l=0}^{L-1} c_{l} \varphi_{m n}\left(t-\tau_{l}\right) e^{j 2 \pi v_{l} t} d t \\
& =\sum_{l=0}^{L-1} c_{l} \int \psi_{00}^{*}(t) \varphi_{m n}\left(t-\tau_{l}\right) e^{j 2 \pi v_{l} t} d t,
\end{aligned}
$$

the intercorrelation between $X$ and $Y_{m n}$ can be expressed as

$$
\begin{aligned}
\mathbb{E} & {\left[X^{*} Y_{m n}\right] } \\
& =\mathbb{E}\left[\sum_{k=0}^{L-1} c_{k}^{*} \sum_{l=0}^{L-1} c_{l} \int \psi_{00}^{*}(t) \varphi_{m n}\left(t-\tau_{l}\right) e^{j 2 \pi v_{l} t} d t\right] \\
& =\sum_{k, l=0}^{L-1} \underbrace{\mathbb{E}\left[c_{k}^{*} c_{l}\right]}_{\pi_{k} \delta_{k l}} \int \psi_{00}^{*}(t) \varphi_{m n}\left(t-\tau_{l}\right) e^{j 2 \pi v_{l} t} d t \\
& =\sum_{k=0}^{L-1} \pi_{k} \int \psi_{00}^{*}(t) \varphi_{m n}\left(t-\tau_{k}\right) e^{j 2 \pi v_{k} t} d t .
\end{aligned}
$$

Hence, we obtain

$$
\begin{aligned}
& \Re\left\{\left(\mathbb{E}\left[X^{*} Y_{m n}\right]\right)^{2}\right\} \\
& =\mathfrak{R}\left\{\left(\sum_{k=0}^{L-1} \pi_{k} \int \psi_{00}^{*}(t) \varphi_{m n}\left(t-\tau_{k}\right) e^{j 2 \pi v_{k} t} d t\right)^{2}\right\} .
\end{aligned}
$$

Since the variance of $X$ is given by $\mathbb{E}\left[|X|^{2}\right]=$ $\mathbb{E}\left[\left(\sum_{k=0}^{L-1} c_{k}\right)^{*}\left(\sum_{l=0}^{L-1} c_{l}\right)\right]=\sum_{k, l=0}^{L-1} \mathbb{E}\left[c_{k}^{*} c_{l}\right]=\sum_{k=0}^{L-1} \pi_{k}$, we can write

$$
\begin{aligned}
& \frac{\mathfrak{R}\left\{\left(\mathbb{E}\left[X^{*} Y_{m n}\right]\right)^{2}\right\}}{\mathbb{E}\left[|X|^{2}\right]} \\
& =\frac{\mathfrak{R}\left\{\left(\sum_{k=0}^{L-1} \pi_{k} \int \psi_{00}^{*}(t) \varphi_{m n}\left(t-\tau_{k}\right) e^{j 2 \pi v_{k} t} d t\right)^{2}\right\}}{\sum_{k=0}^{L-1} \pi_{k}} .
\end{aligned}
$$

As $Y_{m n}=\sum_{k=0}^{L-1} c_{k} \int \psi_{00}^{*}(t) \varphi_{m n}\left(t-\tau_{k}\right) e^{j 2 \pi v_{k} t} d t$, the variance of $Y_{m n}$ is expressed as

$$
\begin{aligned}
\mathbb{E}\left[\left|Y_{m n}\right|^{2}\right]=\mathbb{E} & {\left[\left(\sum_{k=0}^{L-1} c_{k} \int \psi_{00}^{*}(t) \varphi_{m n}\left(t-\tau_{k}\right) e^{j 2 \pi v_{k} t} d t\right)^{*}\right.} \\
& \left.\cdot\left(\sum_{l=0}^{L-1} c_{l} \int \psi_{00}^{*}(s) \varphi_{m n}\left(s-\tau_{l}\right) e^{j 2 \pi v_{l} s} d s\right)\right]
\end{aligned}
$$




$$
\begin{gathered}
=\sum_{k, l=0}^{L-1} \mathbb{E}\left[c_{k}^{*} c_{l}\right] \iint \psi_{00}(t) \psi_{00}^{*}(s) \varphi_{m n}^{*}\left(t-\tau_{k}\right) \\
\cdot \varphi_{m n}\left(s-\tau_{l}\right) e^{j 2 \pi\left(v_{l} s-v_{k} t\right)} d t d s \\
=\sum_{k=0}^{L-1} \pi_{k} \iint \psi_{00}(t) \psi_{00}^{*}(s) \varphi_{m n}^{*}\left(t-\tau_{k}\right) \\
\cdot \varphi_{m n}\left(s-\tau_{k}\right) e^{j 2 \pi v_{k}(s-t)} d t d s \\
=\sum_{k=0}^{L-1} \pi_{k} \iint \psi_{00}(t) \psi_{00}^{*}(s) \varphi_{m n}^{*}\left(t-\tau_{k}\right) \\
\cdot \varphi_{m n}\left(s-\tau_{k}\right) e^{j 2 \pi v_{k}(s-t)} d t d s .
\end{gathered}
$$

Finally, we inject (A.14) and (A.15) in (A.10). Therefore, the final expression of $P_{m n}$ is given by

$$
\begin{aligned}
P_{m n}= & \frac{E\left(\sum_{l=0}^{L-1} \pi_{l}\right)}{4\|\varphi\|^{2}}\left[\sum_{k=0}^{L-1} \frac{\pi_{k}}{\sum_{l=0}^{L-1} \pi_{l}}\right. \\
& \cdot \iint \psi_{00}(t) \psi_{00}^{*}(s) \varphi_{m n}^{*}\left(t-\tau_{k}\right) \varphi_{m n}\left(s-\tau_{k}\right) e^{j 2 \pi v_{k}(s-t)} d t d s \\
& +\Re\left\{\left(\sum_{k=0}^{L-1} \frac{\pi_{k}}{\sum_{l=0}^{L-1} \pi_{l}}\right.\right. \\
& \left.\left.\left.\cdot \int \psi_{00}^{*}(t) \varphi_{m n}\left(t-\tau_{k}\right) e^{j 2 \pi v_{k} t} d t\right)^{2}\right\}\right]
\end{aligned}
$$

\section{B. Conveniently Scaled Version of the Time-Frequency Lattice for FBMC Systems}

In this appendix, we explain the simplified framework used in Section 7, which is based on a convenient scaling of time and frequency axes. We denote by $\Delta=1 / F T$ the lattice density in FBMC (QAM or OQAM) systems. To maximize the SINR, the delay spread and the Doppler spread must be balanced in time and frequency. Hence, we should choose $F$ and $T$ such as $B_{d} / F=T_{m} / T$. Then, for a simplified study and exploration of our system, we start by scaling the time axis by multiplying the time $t$ by $1 / T \sqrt{\Delta}$, which leads to a normalized time spacing equal to $T^{\prime}=1 / \sqrt{\Delta}$ and a normalized delay spread $T_{m}^{\prime}=T_{m} / T \sqrt{\Delta}$. This time scaling leads to a frequency scaling by a multiplication by $T \sqrt{\Delta}$. As a consequence, we end up with a normalized frequency spacing equal to $F^{\prime}=$ $F(T \sqrt{\Delta})=F T \sqrt{\Delta}=1 / \sqrt{\Delta}$, since $F T=1 / \Delta$. The Doppler spread is also scaled by this operation and gives a normalized Doppler spread equal to $B_{d}^{\prime}=B_{d}(T \sqrt{\Delta})$. On the one hand, $B_{d}^{\prime} T_{m}^{\prime}=\left(B_{d}(T \sqrt{\Delta})\right)\left(T_{m} / T \sqrt{\Delta}\right)=B_{d} T_{m}$. On the other hand, thanks to the balanced distribution of Doppler and delay spreads, we have $B_{d} / F=T_{m} / T$. Therefore, $B_{d}^{\prime}=B_{d}(T \sqrt{\Delta})=$ $\left(T_{m} F / T\right)(T \sqrt{\Delta})=T_{m} F T \sqrt{\Delta} / T=T_{m} / T \sqrt{\Delta}=T_{m}^{\prime}$ (as $B_{d}=$ $\left.T_{m} F / T\right)$. Consequently, $B_{d}^{\prime}=T_{m}^{\prime}=\sqrt{B_{d} T_{m}}$. In summary, thanks to scaling, we end up with an equivalent system where $T^{\prime}=F^{\prime}=1 / \sqrt{\Delta}$ and $B_{d}^{\prime}=T_{m}^{\prime}=\sqrt{B_{d} T_{m}}$. This is the model that we use in Section 7 to assess the performances of the systems at hand. It is with these assumptions that we work in practice.

\section{Conflicts of Interest}

The authors declare that there are no conflicts of interest regarding the publication of this paper.

\section{References}

[1] G. Wang, L. Zhuang, and K. Shao, "Time-varying multicarrier and single-carrier modulation systems," IET Signal Processing, vol. 7, no. 1, pp. 81-92, 2013.

[2] T. Yunzheng, L. Long, L. Shang, and Z. Zhi, "A Survey: Several Technologies of Non-Orthogonal Transmission for 5G," China Communications, vol. 12, no. 10, pp. 1-15, 2015.

[3] https://www.metis2020.com/.

[4] http://fantastic5g.com/.

[5] http://www.ict-emphatic.eu/.

[6] http://5gnow.eu/.

[7] G. Wunder, P. Jung, M. Kasparick et al., "5GNOW: nonorthogonal, asynchronous waveforms for future mobile applications," IEEE Communications Magazine, vol. 52, no. 2, pp. 97$105,2014$.

[8] B. Farhang-Boroujeny, "OFDM versus filter bank multicarrier," IEEE Signal Processing Magazine, vol. 28, no. 3, pp. 92-112, 2011.

[9] B. Saltzberg, "Performance of an efficient parallel data transmission system," IEEE Transactions on Communication Technology, vol. 15, no. 6, pp. 805-811, 1967.

[10] K. El Baamrani, V. P. G. Jiménez, A. G. Armada, and A. A. Ouahman, "Multiuser subcarrier and power allocation algorithm for OFDM/Offset-QAM," IEEE Signal Processing Letters, vol. 17, no. 2, pp. 161-164, 2010.

[11] H. Saeedi Sourck, Y. Wu, J. W. M. Bergmans, S. Sadri, and B. Farhang-Boroujeny, "Effect of carrier frequency offset on offset QAM multicarrier filter bank systems over frequency-selective channels," in Proceedings of the IEEE Wireless Communications and Networking Conference 2010, WCNC 2010, April 2010.

[12] H. Lin, M. Gharba, and P. Siohan, "Impact of time and carrier frequency offsets on the FBMC/OQAM modulation scheme," Signal Processing, vol. 102, pp. 151-162, 2014.

[13] Z. Hraiech, F. Abdelkefi, and M. Siala, "POPS-OFDM: Pingpong Optimized Pulse Shaping-OFDM for 5G systems," in Proceedings of the IEEE International Conference on Communications, ICC 2015, pp. 4781-4786, June 2015.

[14] M. Siala, F. Abdelkefi, and Z. Hraiech, "Novel algorithms for optimal waveforms design in multicarrier systems," in Proceedings of the 2014 IEEE Wireless Communications and Networking Conference, WCNC 2014, pp. 1270-1275, April 2014.

[15] R. Razavi, P. Xiao, and R. Tafazolli, "Information theoretic analysis of OFDM/OQAM with utilized intrinsic interference," IEEE Signal Processing Letters, vol. 22, no. 5, pp. 618-622, 2015.

[16] C. Kim, K. Kim, Y. H. Yun, Z. Ho, B. Lee, and J.-Y. Seol, “QAMFBMC: A new multi-carrier system for post-OFDM wireless communications," in Proceedings of the 58th IEEE Global Communications Conference, GLOBECOM 2015, December 2015.

[17] P. Bello, "Characterization of randomly time-variant linear channels," IEEE Transactions on Communications, vol. 11, no. 4, pp. 360-393, 1963. 
[18] P. Blanchard and E. Brüning, "Mathematical methods in physics distributions, hilbert space operators, and variational methods," Progress in Mathematical Physics, vol. 26, 2003.

[19] M. J. Bastiaans, "Application of the WIGner distribution function in optics," in The Wigner Distribution: Theory and Application in Signal Processing, W. Meckelenbräuker and F. Hlawatsch, Eds., pp. 375-426, Elsevier Science, 1997.

[20] M. Abramowitz and I. A. Stegun, Handbook of Mathematical Functions with Formulas, Graphs, and Mathematical Tables, vol. 55 of National Bureau of Standards Applied Mathematics Series55, Dover Publications, Inc., New York, NY, USA, 1964.

[21] S. P. Lloyd, "Least squares quantization in PCM," Institute of Electrical and Electronics Engineers Transactions on Information Theory, vol. 28, no. 2, pp. 129-137, 1982.

[22] L. L. Scharf and C. Demeure, Statistical Signal Processing: Detection, Estimation, and Time Series Analysis, Addison-Wesley Publishing Company, 1991.

[23] Recommendation ITU-R M.1225, Guidelines for Evaluation of Radio Transmission Technologies for IMT-2000, 1997.

[24] B. Le Floch, M. Alard, and C. Berrou, "Coded orthogonal frequency division multiplex," Proceedings of the IEEE, vol. 83, no. 6, pp. 982-996, 1995.

[25] M. Bellanger, "FBMC physical layer: a primer," PHYDYAS Project, 2010.

[26] Deliverable D3.1_v1.1, "5G Waveform Candidate Selection," 5GNOW Project, 2015.

[27] M. Bellili, L. B. H. Slama, and M. Siala, "Multi-pulse/singlepulse design for maximizing SIR in partially equalized OFDM systems over highly dispersive channels," in Proceedings of the 2009 16th IEEE International Conference on Electronics, Circuits and Systems, ICECS 2009, pp. 1004-1007, December 2009. 


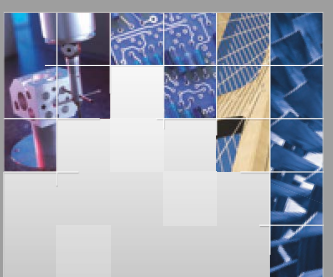

\section{Enfincering}
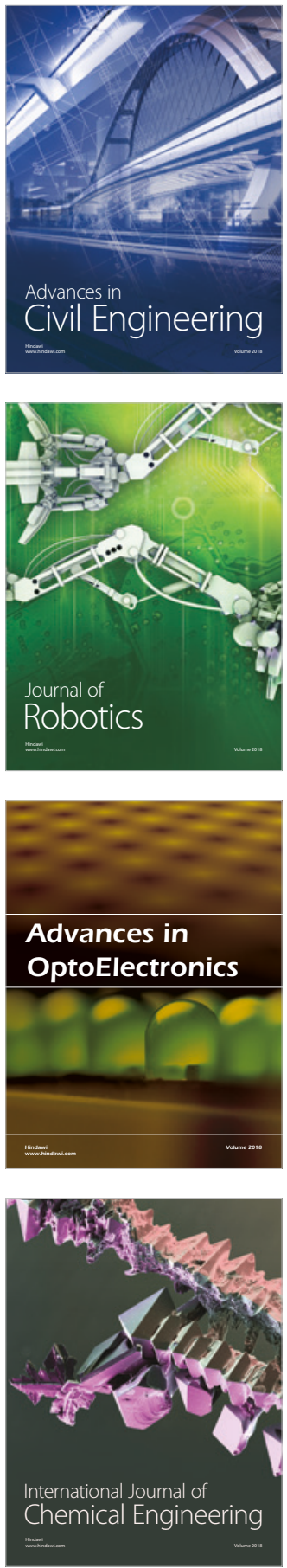

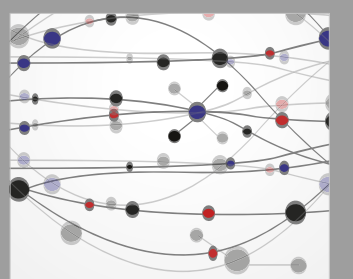

\section{Rotating \\ Machinery}

The Scientific World Journal

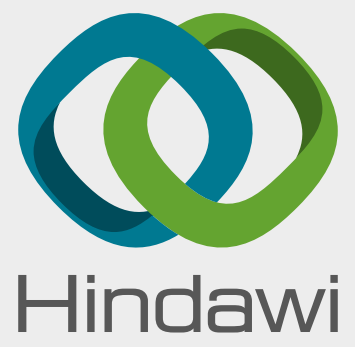

Submit your manuscripts at

www.hindawi.com
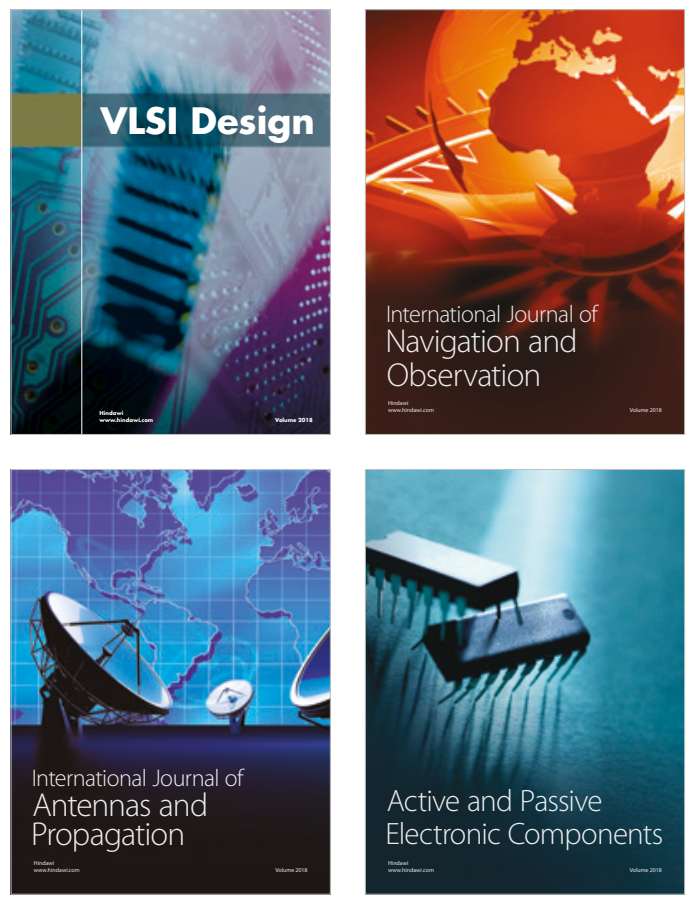
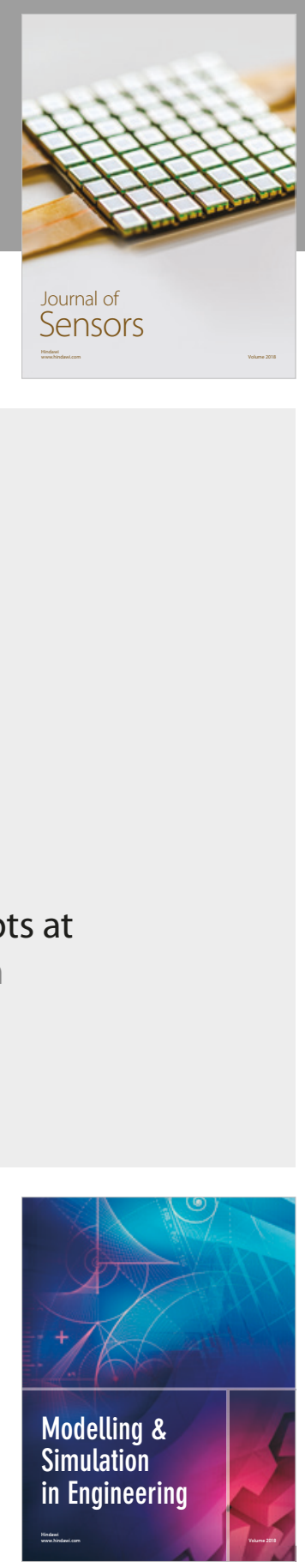

\section{Advances \\ Multimedia}
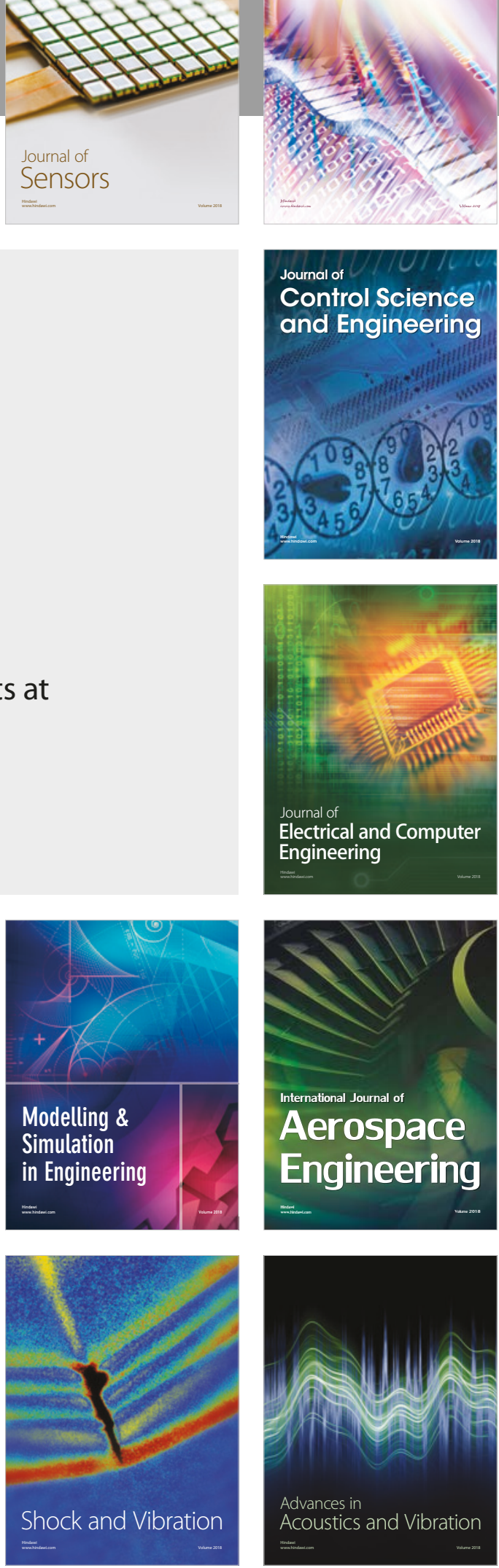